Portland State University

PDXScholar

Spring 6-8-2017

\title{
Predicting Parturition in a Long-Gestating Species: Behavioral and Hormonal Indicators in the Asian Elephant (Elephas maximus)
}

Heather Kelly Velonis

Portland State University

Follow this and additional works at: https://pdxscholar.library.pdx.edu/open_access_etds

Part of the Animal Sciences Commons, Animal Studies Commons, and the Biology Commons Let us know how access to this document benefits you.

\section{Recommended Citation}

Velonis, Heather Kelly, "Predicting Parturition in a Long-Gestating Species: Behavioral and Hormonal Indicators in the Asian Elephant (Elephas maximus)" (2017). Dissertations and Theses. Paper 4021. https://doi.org/10.15760/etd.5905

This Thesis is brought to you for free and open access. It has been accepted for inclusion in Dissertations and Theses by an authorized administrator of PDXScholar. Please contact us if we can make this document more accessible: pdxscholar@pdx.edu. 
Predicting Parturition in a Long-Gestating Species: Behavioral and Hormonal Indicators in the Asian Elephant (Elephas maximus)

by

Heather Velonis

A thesis submitted in partial fulfillment of the requirements for the degree of

Master of Science

in Biology

Thesis Committee:

Deborah A. Duffield, Chair Deborah Lutterschmidt

David Shepherdson

Nadja Wielebnowski

Portland State University 2017 
(C) 2017 Heather Velonis 


\begin{abstract}
Captive populations of Asian elephants (Elephas maximus) in North America are not self-sustaining, and increasing reproductive success within captive populations is a high priority. The ability to accurately predict parturition can have a direct impact on elephant welfare. Elephants in captivity often require significant preparation and management throughout the birthing process, and complications during labor and delivery can necessitate immediate intervention, including stillbirth, protracted labor, maternal aggression towards a newborn calf, and dystocia. Being able to predict when parturition will commence can ensure appropriate staff is available and adequate monitoring is performed. Routine endocrine sampling can be used to predict parturition in Asian elephants, with a drop in progesterone (P4) to baseline levels signaling parturition in 2-5 days. However, we determined this method is not without limitations, and it is not used in all institutions that house elephants. As changes in hormones regulate and alter behaviors, we investigated behavioral indicators as an additional management tool for predicting parturition, a time of drastic hormone changes. We conducted a study of five pregnancies in Asian elephants at the Oregon Zoo, U.S.A, and Taronga Zoo, Australia, between 2008 and 2012.

In Chapter 2, I evaluated progesterone (P4) and cortisol levels across three time periods: Baseline; Pre, (the week preceding the drop in P4); and Post, (the period after the P4 drop). Levels of P4 were significantly lower, and levels of cortisol were significantly higher in the days just prior to parturition. I found considerable intra- and
\end{abstract}


inter-individual variation in both endocrine profiles, which can make endocrine assessments difficult to interpret in real time.

In Chapter 3, I investigated whether behaviors in the preparturition period could be predictive of impending parturition in the Asian elephant. ANOVA results indicated a significant difference in the amount of time that elephants spent walking backwards across three time periods $(F(2)=3.723, p=0.033)$, with the behavior increasing as parturition approached. These results were supported by a non-parametric KruskalWallis. Using a generalized linear mixed model (GLMM), I found that as P4 levels decrease, walking backwards behavior significantly increases.

In Chapter 4, I evaluated investigative trunk behaviors, or "trunk checks", directed towards the temporal gland near the ear, mammary glands, vulva and anus of the pregnant dam. Investigative behaviors included both self-directed behaviors and those sent from herd mates towards the pregnant dam. Self-directed behaviors are most likely associated with physical changes in the pregnant dam, such as using the trunk to pull on swollen teats. Other-directed behaviors may stem from chemo-sensory signaling or other types of communication between herd mates, such as detecting changes in progesterone or cortisol. I ran GLMM and found that four trunk-check behaviors varied significantly with P4 and/or cortisol profiles. These were: self-checks of mammary glands increased with decreasing P4 levels; herd-mate-checks of mammary glands increased with decreasing P4 levels; self-checks of vulva increased with 
decreasing P4 levels and increasing cortisol levels; herd-mate-checks of anus increased with increasing cortisol levels.

In Chapter 5, I evaluated activity budget behaviors in the pregnant elephants. Generalized comparisons were made to published activity budgets of typical captive Asian elephants. I report that activity budgets are within the range of normal activity, though I note a high level of inter-individual variation. In addition, I compared two sampling techniques, including one-zero and instantaneous sampling, that were used for activity budget data collection. I discuss the different results obtained by each sampling technique.

These results are a very promising indication that behaviors, including walking backwards and multiple trunk-check behaviors, are changing over time or with parturition-related hormone profiles. We recommend that keepers, veterinary staff, and other observers that are familiar with the regular behavioral repertoire of a pregnant female should pay close attention to these highlighted behaviors. Keeping track of these behaviors, especially in conjunction with P4 and cortisol tracking, can help staff refine existing windows of expected parturition. 


\section{Acknowledgements}

I am most grateful to my loving family, whose encouragement and support made this journey possible. When I had to work long and unusual hours my wonderful husband, Aquila Velonis, would rearrange his work schedule and take over the primary care of our children. For this, he has earned multiple father-of-the-year awards from me. Our children, Liam and Anwen, injected love and a child's natural curiosity into my research. They often reminded me of my love and admiration for these wonderful animals when I most needed reminding.

I am incredibly thankful for the unfailing support of my advisor, Dr. Debbie Duffield. Throughout the (long!) process of obtaining my degree, Deb has always been available to listen, give advice, and set me back on the path with a smile. She has read countless versions of this thesis, and improved it with thoughtful edits and changes every time. Deb is a treasure!

I am indebted to Karen Lewis and Sharon Glaeser at the Oregon Zoo. Karen shared with me all of her knowledge of elephant behavior in general, and of this preparturition project in particular. We spent countless hours and days reviewing data sets and analyses at the Oregon Zoo. In addition, Karen graciously read numerous draft chapters, and her editing and insight was invaluable. Sharon graciously reviewed endocrine profiles and patterns with me, and we had insightful conversations that helped me to examine my data and results in a new light. 
I am grateful to my committee for their support and input into this project.

Thank you to Dr. Nadja Wielebnowski and Dr. Deborah Lutterschmidt for providing much needed insight and clarification on all things endocrine-related, and to Dr. David Shepherdson for providing similar insight and clarification on all things animal behaviorrelated.

Thank you to everyone who made this thesis possible. 


\section{Table of Contents}

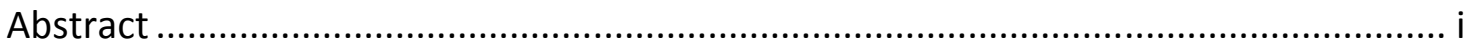

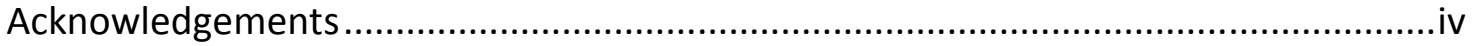

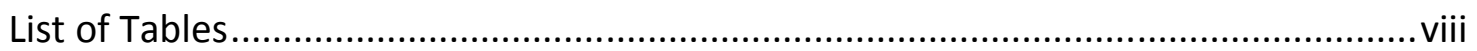

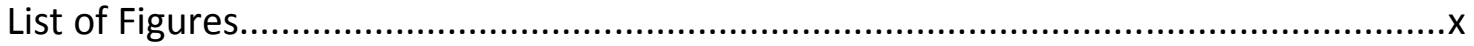

Chapter 1: Introduction and General Methodology ........................................................... 1

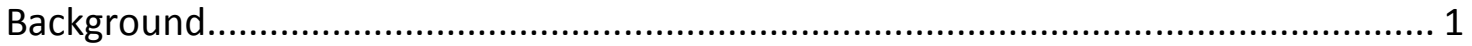

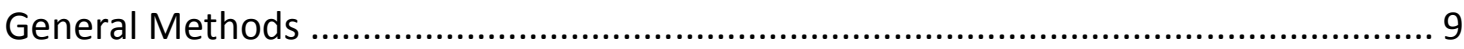

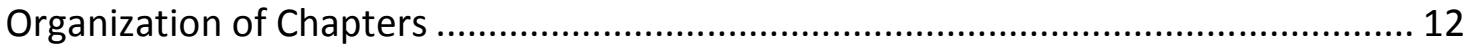

Chapter 2: Endocrine Profiles in Gestating Asian Elephants ..................................................17

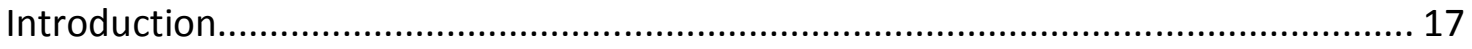

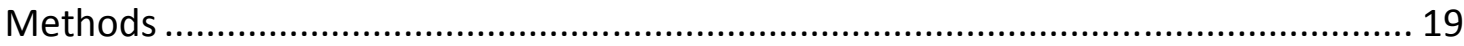

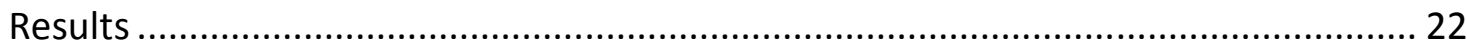

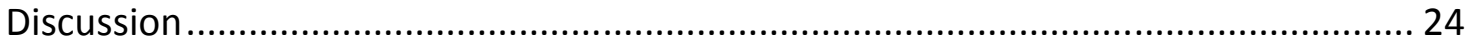

Chapter 3: Preparturition Behaviors as Predictive Tools in Late Gestation ................................ 32

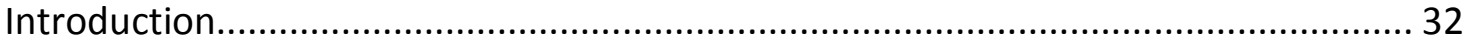

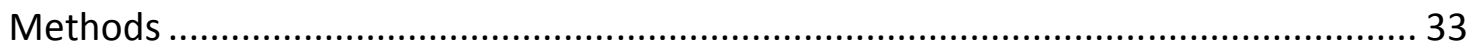

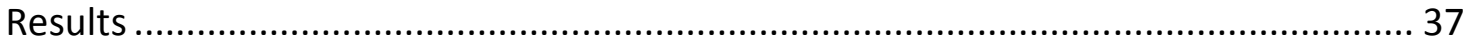

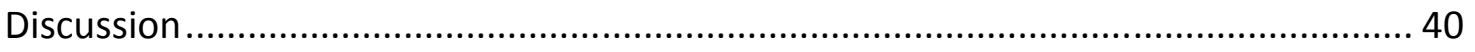

Chapter 4: Trunk-Check Behaviors as Predictive Tools in Late Gestation ................................... 47

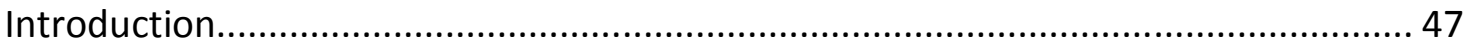

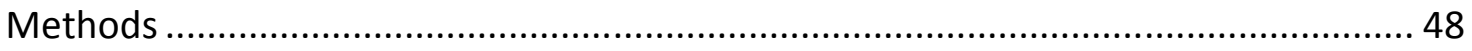

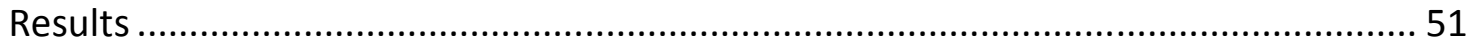

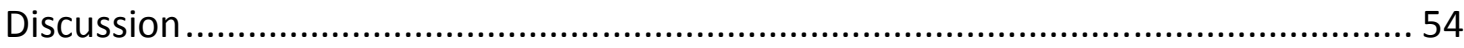

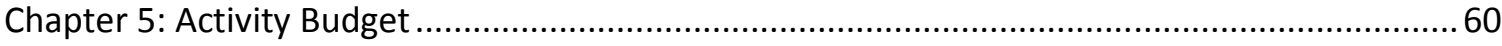

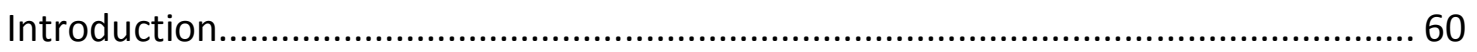

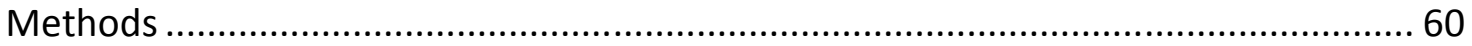

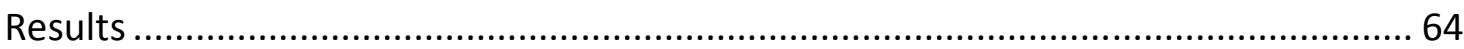

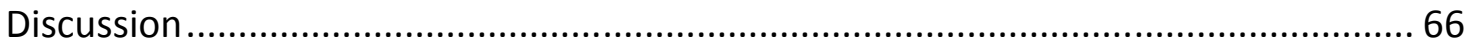




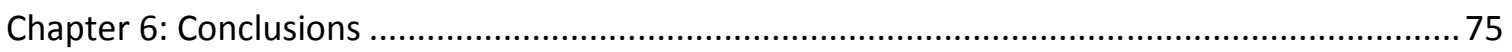

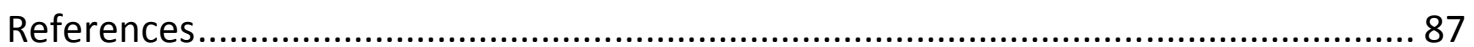




\section{List of Tables}

Table 1.1 Birth protocol documentation of behaviors observed prior to parturition...... 15

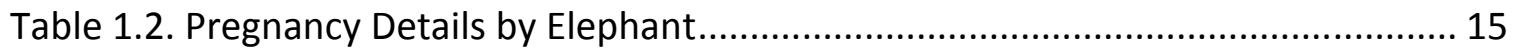

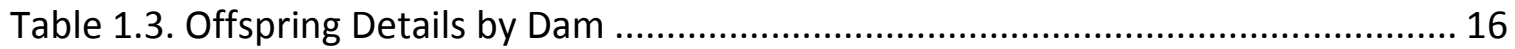

Table 1.4. Reliability Test Index of Concordance for Oregon Zoo, 2012, By Observer .... 16

Table 2.1. Schedule of Blood Draws for Duration of Gestation, By Elephant .................. 31

Table 2.2. Day Count of Records Used in Preparturition Analysis.................................. 31

Table 3.1. Ethogram Variables for Preparturition Behaviors....................................... 43

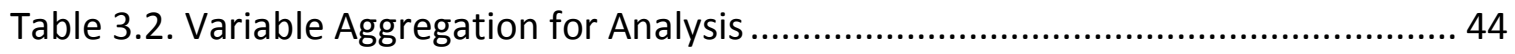

Table 3.3. Likelihood Ratio Test for Preparturition Behaviors as a function of Partitioned

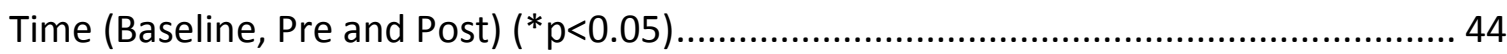

Table 3.4. ANOVA: Preparturition Behaviors in Baseline, Pre and Post Time Periods, With

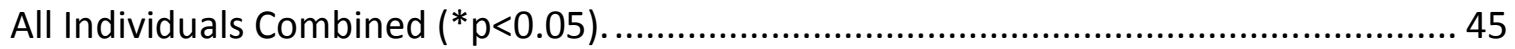

Table 3.5. Post-Hoc Comparisons Between Baseline, Pre \& Post periods, With All

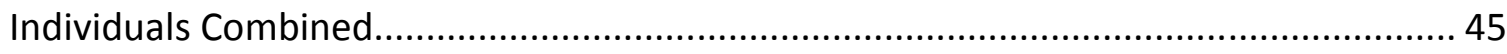

Table 3.6. Kruskal-Wallis Preparturition Behaviors as a Function of Partitioned Time

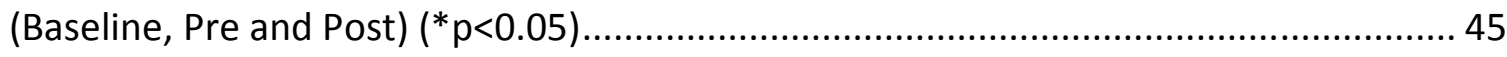

Table 3.7. Variable Loadings by Component (highest loadings in bold)......................... 46

Table 3.8. GLMM PCA Body Change Component with Data Partition, P4, and Cortisol as

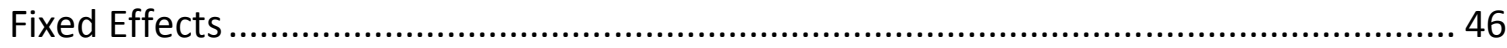

Table 3.9. GLMM Models with Body Change Component and Each Fixed Effect............ 46

Table 3.10. GLMM Walking Backwards (Best Fit Model in Bold) .................................. 46

Table 4.1. Ethogram Variables for Trunk-Check Behaviors ........................................ 56

Table 4.2. Likelihood Ratio Test for Trunk-Check Behaviors as a function of Partitioned Time (Baseline, Pre \& Post periods)...................................................................... 56

Table 4.3. ANOVA: Trunk-Check Behaviors in Baseline, Pre and Post time periods, with

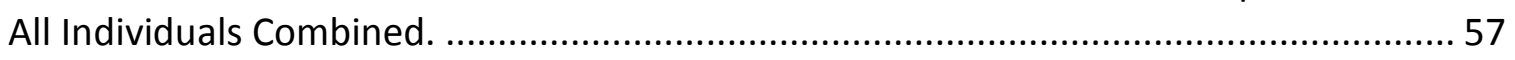

Table 4.4. Kruskal-Wallis Trunk Check Behaviors as a Function of Data Partition ........... 57

Table 4.5. Trunk-Check Variable Loadings by Component (highest loadings in bold). .... 57

Table 4.6. GLMM PCA Component 1 with Data Partition, P4, and Cortisol as Fixed Effects

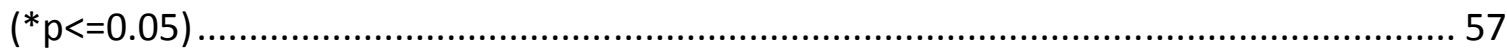


Table 4.7. GLMM Models with PCA Component Vulva-Self and Each Fixed Effect

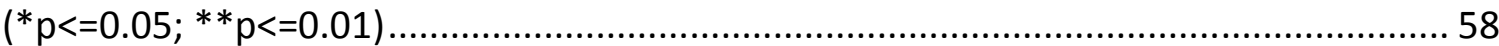

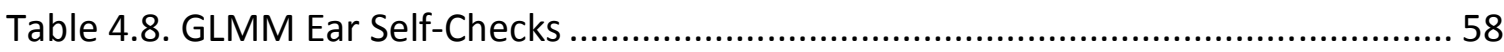

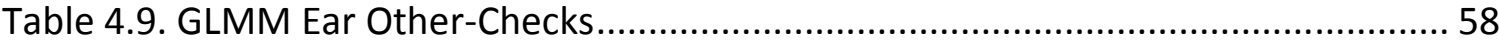

Table 4.10. GLMM Mammary Gland Self-Checks ( $\left.{ }^{*} p<0.05\right)$ (Best Fit Model in Bold)..... 58

Table 4.11. GLMM Mammary Gland Other-Checks ( ${ }^{*} p<0.05, * * p<0.01$ ) (Best Fit Model

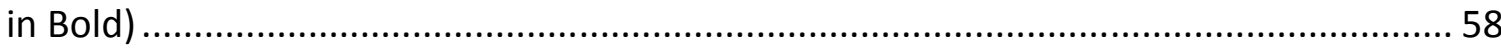

Table 4.12. GLMM Vulva Self-Checks $\left({ }^{*} p<0.05,{ }^{* *} p<0.01\right)$ (Best Fit Model in Bold)...... 59

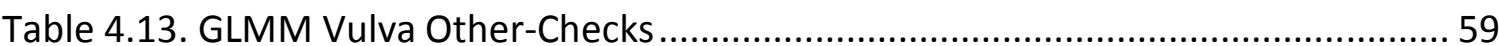

Table 4.14. GLMM Anus Other-Checks (*p<0.05, $\left.{ }^{* *} p<0.01\right)$ (Best Fit Model in Bold) .... 59

Table 5.1. Ethogram of Activity Budget Behaviors .......................................................... 70

Table 5.2. Variable aggregation for analysis of data collected using one-zero sampling 71

Table 5.3. Variable aggregation for analysis of data collected using instantaneous

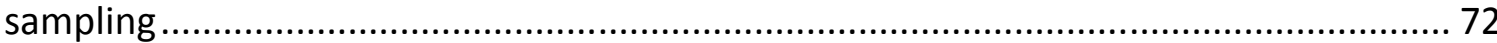

Table 5.4. Count of Records Used in Activity Budget Analysis ...................................... 73

Table 5.5. Average daily occurrence of each behavioral category (One-Zero Sampling). 73

Table 5.6. All behavioral categories showed significant mean differences between at least two elephants $\left({ }^{* *} p<0.01\right)($ One-Zero) .................................................................... 73

Table 5.7. Tukey's HSD mean difference scores and significance levels ( ${ }^{*} p<0.05$;

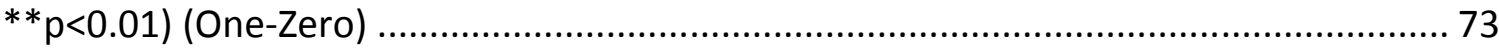

Table 5.8. Average number of occurrences observed using instantaneous sampling ..... 74

Table 5.9. Locomotion and Feeding behavioral categories showed significant mean differences between at least two elephants $\left({ }^{*} p<0.05,{ }^{* *} p<0.01\right)$ (Instantaneous) ......... 74

Table 5.10. Tukey's HSD mean difference scores and significant levels ( ${ }^{*} p<0.05$;

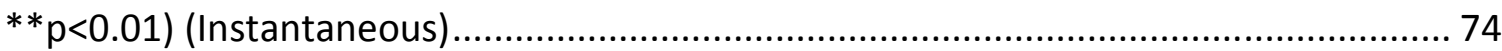




\section{List of Figures}

Figure 1.1. Distribution of Asian Elephant Populations in 2011(Credit: Riccardo Pravettoni , cartographer/designer, GRID-Arendal; http://www.grida.no/graphicslib/detail/asian-elephant-population-estimates_478d).. 14

Figure 2.1. Progesterone data partitioned into Baseline, Pre and Post time periods for a representative animal from the study.

Figure 2.2. Individual profiles show a decrease in $\mathrm{P} 4$ levels in the days prior to parturition for (a) Rose-Tu 2008; (b) Rose-Tu 2012; (c) Pak Boon; (d) Porntip; (e) Thong Dee.

Figure 2.3. Late gestation progesterone profiles show a decrease in P4 concentration in the days prior to parturition for all elephants ............................................................ 29

Figure 2.4. Late Gestation Cortisol Profiles ............................................................. 29

Figure 2.5. Late Gestation Progesterone Profiles Reported as a Percentage of Each

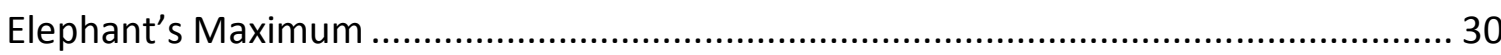

Figure 2.6. Late Gestation Cortisol Profiles Reported as a Percentage of Each Elephant's

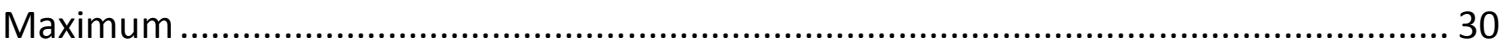

Figure 5.1. Average daily proportion of intervals during which each behavior was observed (one-zero sampling). 69

Figure 5.2. Average daily proportion of time observed in each behavior category (instantaneous sampling). 


\section{Chapter 1: Introduction and General Methodology}

\section{Background}

Captive populations of Asian elephants (Elephas maximus) in North America are not self-sustaining due to poor reproductive success (Wiese 2000; Rees 2003; Brown et al. 2004b, Wiese \& Willis 2004). Both low fecundity and higher than average rates of calf death reduce the long-term viability of maintaining Asian elephants in the captive setting (Taylor \& Poole 1998; Wiese 2000; Rees 2003; Brown et al. 2004b; Szdzuy et al. 2006). Increasing reproductive success within captive populations is a high priority (Taylor \& Poole 1998; Brown et al. 2004a), especially with decreasing feasibility of supplementing herds with imported and wild-caught individuals due to political and legal barriers (Hildebrandt et al. 2006; Hutchins \& Keele 2006).

The ability to accurately predict parturition can have a direct impact on elephant welfare. Elephants in captivity require significant preparation and management throughout the birthing process (Kowalski et al. 2010). This is especially true of inexperienced individuals who have neither given birth nor observed parturition in a herd-mate. Complications during labor and delivery can require immediate intervention, including stillbirth, protracted labor, maternal aggression towards a newborn calf (Taylor \& Poole 1998; Szdzuy 2006), and in rare cases, dystocia following retention of a dead fetus (Thitaram et al. 2006; Schaftenaar 2013). Institutions with successful breeding programs, including the Oregon Zoo, Taronga Zoo, Riddle's Elephant and Wildlife Sanctuary, and Disney's Animal Kingdom, employ detailed birth protocols to 
address these management issues. According to these protocols, preparations can include habituating elephants to routine blood draws, performing physical manipulations (such as teat pulling to prepare the dam for a nursing calf), managed exercise routines, and "around-the-clock" visual monitoring once parturition is imminent. The ability to predict when parturition will commence can ensure appropriate staff is available and adequate monitoring is performed (Szdzuy et al. 2006). Routine endocrine sampling is used by many Association of Zoos and Aquariums (AZA) institutions to monitor estrous cycles, to detect or confirm pregnancy, and to predict parturition. Based on responses to a 2001-2002 Elephant Taxon Advisory Group (TAG)/Species Survival Plan (SSP) reproductive survey, approximately $49 \%$ of Asian and $62 \%$ of African elephants in North America undergo weekly endocrine monitoring for estrous cyclicity (Brown et al. 2004b). The use of ultrasound technology to confirm pregnancy and hormone data to predict parturition in elephants has been described in detail and incorporated into birth protocols for decades (Schmidt 1982; Hess et al. 1983; Hodges et al. 1999; Dehnhard et al. 2003). In pregnant dams, a significant drop in serum concentrations of progesterone (P4) is used to predict parturition, which typically occurs 2-5 days after baseline levels are observed (Hess et al. 1983; Hodges et al. 1999; Dehnhard et al. 2003, Meyer et al. 2004). These methods provide a very short window at the end of a long and highly variable gestation that can last up to 23 months (Hildebrandt et al. 2006; Dale et al. 2010). However, due to intra-individual daily fluctuations and inter-individual variation accurately detecting a drop in P4 can be 
challenging in real-time conditions (Sharon Glaeser pers. comm. 2016). Thus, additional predictive tools would be useful not only to organizations that do not have regular endocrine assessments, but to organizations that, despite performing endocrine analyses, have missing or unreliable data, or need to support endocrine sampling timelines. Management tools that can assist in anticipating the birthing process would be greatly valued.

Because changes in hormones regulate and alter behaviors, we have investigated behavioral indicators as a possible management tool for predicting parturition, a time of drastic hormone changes. While many preparturition behaviors are anecdotally mentioned in elephant birth protocols, few behavioral data have undergone thorough examination. The goal of this study was to examine preparturient behaviors and hormone levels in nulliparous and primiparous pregnant captive Asian elephants in order to determine whether behaviors can aid in predicting parturition in Asian elephants.

\section{Asian Elephant Life History}

Elephants are the largest currently-living land mammal. Asian elephants are sexually dimorphic in size (Sukumar 2003), with captive males standing up to $2.7 \mathrm{~m}$ tall and weighing $5400 \mathrm{~kg}$, and captive females standing up to $2.35 \mathrm{~m}$ tall and weighing between $2720 \mathrm{~kg}$ and $4160 \mathrm{~kg}$ (Owen-Smith 1988). Wild populations are estimated between approximately 38,000 and 52,000 individuals, with an additional 16,000 in captivity in Asia (Sukumar 2006). In North America, there are approximately 252 Asian 
elephants in captivity (Keele 2015). Their historical range was throughout Asia, with current wild and semi-captive populations distributed in isolated pockets throughout India, Nepal, and Southeast Asia (Figure 1.1). Asian elephants inhabit a wide range of tropical forests from wet to dry, and low to high elevation. In semi-captive labor camps, Asian elephants have been reported to live over 70 years, but the typical elephant in these conditions is more likely to have a lifespan closer to 30 years (Wiese \& Willis 2004). In captive settings in North America, Asian elephants have an average life expectancy of 44.8 years (Wiese \& Willis 2004).

Elephants are highly social animals, with related females and their juveniles forming multigenerational groups (Moss 1988; Vidya \& Sukumar 2005) of generally three to eight individuals (Sukumar 2003; De Silva et al. 2011). In a group, the eldest female is the matriarch of the herd, leading and setting the pace of daily life. Recent work by De Silva et al. (2011) has shown that like African elephants, Asian elephants will form temporary, multi-group herds, as well as break into smaller daughter-led herds, in a fission-fusion dynamic social system. Males are ousted from the herd between 10 and 15 years of age, around the time they reach puberty or sexual maturity, and form loosely bound bachelor herds or remain solitary, except when breeding (Sukumar 1989; Vidya \& Sukumar, 2005).

Females reach sexual maturity in their early teens, with captive females remaining reproductive into their thirties (Taylor \& Poole 1998; Wiese 2000), while wild and semi-wild females may remain reproductive for longer periods of time (Sukumar 
1989; Taylor \& Poole 1998; Sukumar 2003). Gestation is the longest of any land mammal, and can range from 20 to 23 months, with an average of 658 days (Hildebrandt et al. 2006; Dale et al. 2010). Females typically give birth to a single calf, with an average birthing interval of about four years (Sukumar 1989). Newborn calves are taken care of by their mother, as well as by other adult females in the group known as allomothers or "aunties" (Sukumar 2003).

As highly intelligent animals with complex social systems, elephants have developed numerous forms of communication, including tactile, visual, acoustic and chemical communication. In tactile communication, body rubbing is often observed, and trunks are commonly used for greeting and investigating other elephants (Sukumar 2003). Though they have relatively poor vision, elephants communicate with visual displays of aggression and courtship (Sukumar 2003). Acoustic communication plays a larger role, with elephants producing a wide range of sounds, from infrasonic, low frequency sound waves at $10 \mathrm{~Hz}$ up to about $9000 \mathrm{~Hz}$ (Payne et al. 1986; Vidya \& Sukumar 2005). Male and females also signal their reproductive state with musth and estrous rumbles, respectively (Sukumar 2003). Chemical communication is used to signal reproductive states, with a range of chemical compounds detected in temporal gland secretions, urine, and fecal matter (Sukumar 2003).

\section{Conservation Status}

The International Union for Conservation of Nature (IUCN) lists Asian elephants as endangered, with population trends decreasing (The IUCN Red List of Threatened 
Species 2016). According to the Asian Elephant Conservation Act (2002), the greatest threat to Asian elephants is habitat loss, largely from logging and expansion of agriculture. The loss of habitat is exacerbated by the fact that many Asian elephant populations live in regions with dense human populations (Sukumar 2006). This leads to increased human-elephant interaction and conflict. Conservation work is focused on reducing habitat loss, increasing education, and working with human populations that are impacted by elephant destruction of agriculture (Asian Elephant Conservation Act 1999-2001).

\section{Preparturition Behaviors}

Many species exhibit behavioral changes prior to giving birth. Some of these behaviors are referred to as "nesting" behaviors, as the pregnant female prepares for the arrival of her newborn. Pigs watched for 72 hours pre-parturition showed increased activity as parturition approached (Hartsock e $t$ al.1997). In the 24 hours before the delivery of the first pig, sows changed positions, drank, urinated, defecated, rooted, and pawed the floor more frequently than in the previous two days. Chaloupková and associates (2011) also observed nest building behaviors in the 24 hours prior to delivering the first piglet.

New Zealand white rabbits showed significant behavioral changes in the days preceding parturition (Gonzalez-Mariscal et al. 1994). Digging behavior commenced between 8 to 6 days preparturition. As digging behavior declined, the onset of straw carrying occurred between day 3 to 1 preparturition. This was followed by hair pulling, 
which completed the construction of the nest. On days 2 to 1 preparturition, the pregnant females significantly reduced food intake. The nest-building behaviors in the preparturient period were compared to levels of the hormones beta-estradiol, progesterone, prolactin and beta-endorphin (Negatu \& McNitt 2002). Notably, betaestradiol increased as parturition approached, with a significant increase 2 days preparturition. Progesterone decreased throughout pregnancy, but a significant decrease occurred 2 days preparturition. Prolactin showed a significant increase in concentration on the last day of pregnancy.

In rhesus monkeys and Japanese macaques, pregnant females showed behavior changes prior to parturition associated with changes in hormones (Bardi et al. 2003), as well as increased withdrawal from social life as gestation progressed, paralleling prepartum increases in estrone conjugates. In addition, grooming behaviors shifted prior to parturition with mate-grooming being replaced by self-grooming. This shift in behavior paralleled variations in the ratio of estrone conjugates to pregnanediolglucuronide.

Early observations of captive Asian and African elephants highlighted behaviors in the preparturition period (Maberry 1963; Lang 1967; Styles 1982; Rietkerk et al. 1993). Szdzuy et al. (2006) evaluated night-time behaviors and endocrine profiles. They reported an increase in stereotypic behavior, restless activity, tail beating against the vulva, and throwing hay and sand against the belly in the preparturition period. Progesterone was also reported to decrease to baseline levels 2-3 days prior to 
parturition. Previous work has examined the link between reproductive hormones and expressed behaviors in elephants. Slade-Cain et al. (2008) report that the estrous state of a captive female elephant influences the rate of certain behaviors, including investigative trunk behaviors, aggression, and tail flicking.

In addition to these studies, a review of veterinary guidelines (Schaftenaar \& Hildebrandt 2006) and elephant birth protocols from Oregon Zoo, Taronga Zoo, Riddle's Elephant and Wildlife Sanctuary, and Disney's Animal Kingdom, all institutions with successful breeding programs, revealed common behaviors observed prior to parturition (Table 1.1). Given the common behavioral themes observed in prior studies and at various institutions during the preparturition period, we proposed analyses of selected behaviors to determine if behaviors, either alone or in conjunction with endocrine profiles, can accurately predict parturition. To address this, I had the following specific aims:

(1) Obtain endocrine samples from regularly scheduled blood draws in pregnant elephants to verify the expected patterns in progesterone and cortisol profiles.

(2) Determine whether pre-parturition behavior patterns changed as parturition neared, and/or whether these behaviors change with varying hormone levels.

(3) Determine whether investigative trunk-check behaviors performed by the pregnant dam, as well as by herd mates towards the pregnant dam, change 
as parturition nears, and/or whether these behaviors change with varying hormone levels.

(4) Observe and summarize activity budget behaviors of pregnant elephants. Each of the above specific aims is addressed in the following four chapters.

\section{General Methods}

In this chapter, I address general methodology that applies to the objectives presented in Chapters 2 through 5. Any methodologies specific to a particular objective will be addressed in the respective chapter.

\section{Study Sites, Animals and Husbandry}

Four pregnant Asian elephants were used for this study, one at the Oregon Zoo in Portland, Oregon, U.S.A., and three at the Taronga Zoo in Sydney, Australia, (Table 1.2). At the Oregon zoo, the pregnant female elephant was observed for both her first (nulliparous) and second (primiparous) pregnancies. During her nulliparous pregnancy, she was allowed free access to two older, nulliparous female herd mates, with limited access to three males. During her primiparous pregnancy, her herd structure was identical with the exception that her juvenile male offspring was housed with her. At the Taronga zoo, three elephants were observed during their first (nulliparous) pregnancies. Pregnant females at the Taronga zoo were allowed free access to three other female herd mates during the day, and limited access to the male, who was housed separately. After the first observed pregnancy and successful delivery, the herd structure included a 
juvenile male. After the second observed pregnancy and successful delivery, the herd structure included an additional juvenile male.

Table 1.2 summarizes pregnancy details by elephant (including corresponding North American Studbook and Australasian Species Management Program, or ASMP, identification numbers for each elephant). Details include conception type, maternal age and parity at conception, and the length of gestation for each pregnant female included in the study. The offspring at both institutions were born between 2009 and 2012 and included both male and female calves, with weights at birth ranging from 96 $\mathrm{kg}$ to $136 \mathrm{~kg}$ (Table 1.3).

\section{Hormones}

Both progesterone (P4) and cortisol were selected for evaluation as Oregon and Taronga Zoos routinely monitored these values in their pregnant elephants. Other hormones including prolactin and relaxin and the protein ceruloplasmin were considered for evaluation, but due to limited sample quantities the zoos involved in this study decided not to pursue further hormone analyses at this time.

\section{Behaviors}

An ethogram and check sheet were developed at the Oregon Zoo to monitor the occurrence of the preparturition behaviors of the pregnant dam, investigative trunkcheck behaviors of both self and others, and daily activity budgets. The selected behaviors were based on anecdotal evidence in birth protocols, conversations with zoo keepers and staff, initial observation of the Oregon Zoo elephant, and literature 
searches (Maberry 1963; Lang 1967; Styles 1982; Rietkerk et al. 1993; Szdzuy et al. 2006;

Slade-Cain et al. 2008; Rees 2009; Horback et al. 2014). The ethogram, check sheet and protocol were provided to the Taronga Zoo.

\section{Data Collection}

Behavior observations of each elephant began by approximately day 600 of gestation and continued until the calf was delivered. There were four scheduled, 30minute observations per day, seven days per week. A 30-minute observation was scheduled to begin and end in each of the following 2-hour windows: 9:00-10:59; 11:0012:59; 13:00-14:59; and 15:00-17:00. Each 30-minute observation consisted of $152-$ minute observation intervals. An all-occurrences sampling method was used for all preparturition and investigative trunk-check behaviors (Martin \& Bateson 2007). Both instantaneous and one-zero sampling methods were used for activity budgets (Martin \& Bateson 2007) and are discussed in greater detail in chapter 5.

\section{Observer Training and Reliability at the Oregon Zoo}

All observers at the Oregon Zoo were volunteers. For the 2012 pregnancy at the Oregon Zoo, they were required to attend a training session during which they were provided detailed descriptions of all the behaviors included in the ethogram. Video examples of many of the harder-to-recognize behaviors were reviewed. After the training session, observers were provided training videos, and if desired, live observation training to test their recognition of the behaviors. Each observer was then required to complete a reliability test using the ethogram check sheet during a 30 
minute video created by Oregon Zoo staff. The video compiled a number of the desired behaviors from the ethogram. Each observer's results were evaluated and scored, and an index of concordance (i.e. inter-observer reliability) was determined. In order to avoid over-inflating the indices of concordance, a value of 1 point was applied to observed behaviors, while a lesser value of 0.25 points was applied to blanks representing the absence of behavior. At the Oregon Zoo in 2012, seven observers completed the reliability test with an average index of concordance of $91 \%$, with all observers scoring greater than $85 \%$ (Table 1.4). A similar protocol was required at the Oregon Zoo for the 2008 pregnancy, as well as for all observed pregnancies at the Taronga Zoo. At both institutions, observers were required to pass the reliability test with an index of concordance of at least $80 \%$ to be able to participate in study observations.

\section{Organization of Chapters}

In the following chapters, I will address each of the four main aims and conclusions drawn from this study. In Chapter 2, I discuss the use of endocrine profiles in assessing and monitoring pregnancy status in elephants using regularly scheduled blood draws in pregnant elephants to verify the expected patterns in progesterone and cortisol profiles. In Chapter 3, I look at the need for behavioral indicators as an additional tool in anticipating parturition in Asian elephants. I review previous studies that examine behaviors in the preparturient period, and discuss our selection of preparturition behaviors for study. I address my second aim which was to determine 
whether preparturition behavior patterns changed as parturition neared, and/or whether these behaviors change with varying hormone levels. In Chapter 4, I discuss investigative trunk-check behaviors, and their role in assessing the environment and chemo-signaling between elephants. I address my third aim in which I determine whether investigative trunk-check behaviors performed by the pregnant dam, as well as by herd mates towards the pregnant dam, change as parturition nears, and/or whether these behaviors change with varying hormone levels. In Chapter 5, I evaluate activity budget behaviors to address my fourth aim. I make general comparisons of daily patterns of behavior in late gestation to published typical daily activity patterns of nonpregnant elephants. Finally, in Chapter 6, I discuss conclusions drawn from this study, and address future directions for this work. 


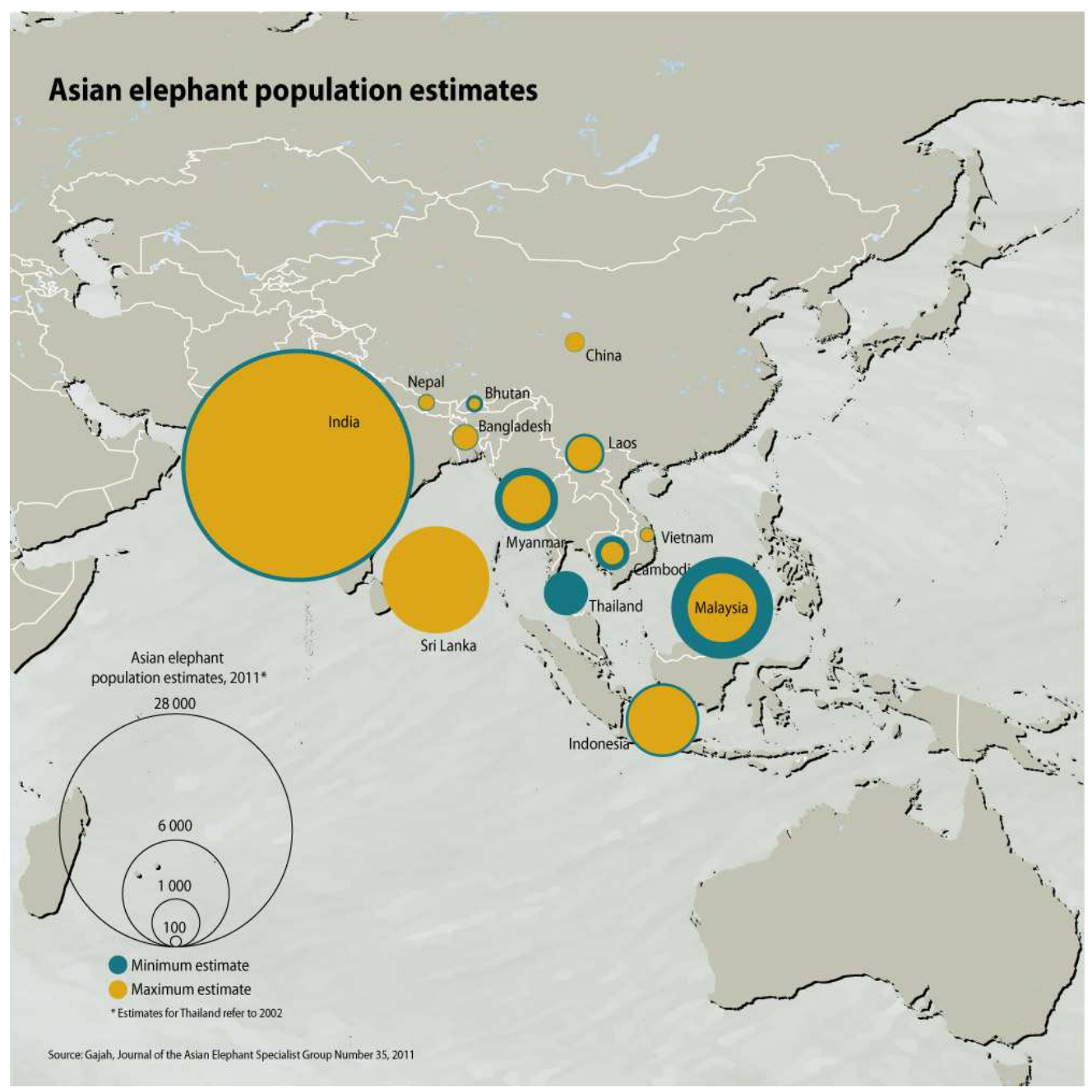

Figure 1.1. Distribution of Asian Elephant Populations in 2011(Credit: Riccardo Pravettoni , cartographer/designer, GRID-Arendal; http://www.grida.no/graphicslib/detail/asian-elephantpopulation-estimates_478d) 


\section{Table 1.1 Birth protocol documentation of behaviors observed prior to parturition}

\section{Several weeks prior to parturition}

Signs of discomfort, including stretching of the body, lordosis and pressing the hind legs backwards against a wall; Abnormal hind limb gait, kneeling down, and climbing; Night pacing; Throwing objects at the belly; Beating the vulva with the tail; Increased or changed behavior of herd mates

\section{Subtle signs of labor}

Restlessness, agitation, aggression, general discomfort, loss of appetite, abnormal posture, freezing in position, tail swishing, leaning into walls, rocking, placing the trunk in mouth, flapping ears more frequently, holding head down, crossing legs, shuffling, standing alone, and investigating urine.

\section{More obvious signs of labor}

Lying down and getting up frequently, kicking at abdomen, frequent defecation and/or urination, onset of colostrum dripping, pulling teats, and swollen vulva; self-exercising at night using rocks, gates or other structures to climb up with hind legs

\section{Marked signs of labor}

Straining, frequent stretching, obvious abdominal contractions, bending knees, vocalization, head stands, going down on one knee, squatting, and bloody discharge; development of a bulge beneath the tail

Table 1.2. Pregnancy Details by Elephant

\begin{tabular}{lllllll}
\hline Institution & Elephant & $\begin{array}{l}\text { Studbook/ } \\
\text { ASMP } \\
\text { Number }\end{array}$ & $\begin{array}{l}\text { Conception } \\
\text { Type (Natural } \\
\text { Breeding or } \\
\text { Artificial } \\
\text { Insemination) }\end{array}$ & $\begin{array}{l}\text { Age at } \\
\text { Conception } \\
\text { in Years } \\
\text { (*Estimate) }\end{array}$ & $\begin{array}{l}\text { Parity at } \\
\text { Conception }\end{array}$ & $\begin{array}{l}\text { Length of } \\
\text { Gestation } \\
\text { Days, } \\
\text { (Months) }\end{array}$ \\
\hline Oregon Zoo & Rose Tu & 361 & Natural & 12 & Nulliparous & $628,(20.6)$ \\
Taronga Zoo & Pak Boon & 62 & Natural & 16 & Primiparous & $647,(21.3)$ \\
& Porntip & 63 & Natural & $15^{*}$ & Nulliparous & $659,(21.7)$ \\
& Thong Dee & 60 & Artificial & $15^{*}$ & Nulliparous & $652,(21.4)$ \\
& Natural & $11^{*}$ & Nulliparous & $654,(21.5)$ \\
\hline
\end{tabular}


Table 1.3. Offspring Details by Dam

\begin{tabular}{llllll}
\hline Institution & Elephant & $\begin{array}{l}\text { Parity at } \\
\text { Conception }\end{array}$ & $\begin{array}{l}\text { Sex of } \\
\text { Offspring }\end{array}$ & $\begin{array}{l}\text { Weight at birth } \\
\text { in kg (pounds) }\end{array}$ & $\begin{array}{l}\text { Year } \\
\text { Delivered }\end{array}$ \\
\hline Oregon Zoo & Rose Tu & Nulliparous & Male & $130(286)$ & 2008 \\
& & Primiparous & Female & $136(300)$ & 2012 \\
Taronga Zoo & Pak Boon & Nulliparous & Female & $120(265)$ & 2010 \\
& Porntip & Nulliparous & Male & $116(256)$ & 2010 \\
& Thong Dee & Nulliparous & Male & $96(212)$ & 2009 \\
\hline
\end{tabular}

Table 1.4. Reliability Test Index of Concordance for Oregon Zoo, 2012, By Observer

\begin{tabular}{lc}
\hline & Index of Concordance \\
\hline Observer 1 & $87 \%$ \\
Observer 2 & $94 \%$ \\
Observer 3 & $93 \%$ \\
Observer 4 & $88 \%$ \\
Observer 5 & $88 \%$ \\
Observer 6 & $93 \%$ \\
Observer 7 & $93 \%$ \\
Average & $91 \%$ \\
\hline
\end{tabular}




\section{Chapter 2: Endocrine Profiles in Gestating Asian Elephants}

\section{Introduction}

Endocrine sampling is commonly used to assess pregnancy status and predict imminent parturition in Asian elephants (Meyer et al. 2004; Oliveira et al. 2008; Kajaysri \& Nokkaew 2014). Predicting elephant parturition using hormone data and ultrasound technology has been described in detail and incorporated into birth protocols for decades (Schmidt 1982; Hess et al. 1983; Hodges et al. 1999; Dehnhard et al. 2003). Routine endocrine sampling is used by many AZA institutions to monitor estrous cycles, to detect or confirm pregnancy, and to predict parturition. At the Oregon and Taronga Zoos, blood draws are taken in the morning hours. Draws typically start weekly in early pregnancy, and increase in frequency as parturition nears, often occurring daily or twice daily in the final days of gestation. A similar schedule is followed by Taronga Zoo.

In the luteal phase of the estrous cycle, the corpus luteum $(\mathrm{CL})$ acts as a temporary endocrine organ, generating progesterone to prepare the uterus for possible implantation of the conceptus (Senger 2003). If conception has not occurred, the CL will degenerate, and progesterone production from the temporary endocrine organ will cease. If conception has occurred, however, the CL will continue to produce progesterone to support the developing pregnancy (Senger 2003). In many species, as the pregnancy progresses progesterone production will shift from the $\mathrm{CL}$ to the placenta, another temporary endocrine organ (Senger 2003; Lueders et al. 2012). However, recent work in the Asian elephant has shown the placenta to be 
steroidogenically inactive (Lueders at al. 2012). Instead, P4 is derived from the CL throughout pregnancy, in part due to CL maintenance from placental lactogen (Yamamoto et al. 2011; Lueders et al. 2012; Yamamoto et al. 2012). There is also evidence that some maternal circulating P4 may be derived from fetal gonads in late gestation (Lueders et al. 2012). Thus, elevated levels of progesterone are used by staff to determine that a pregnancy has occurred, and to monitor the dam to determine if the pregnancy is being maintained.

Levels of progesterone remain elevated throughout pregnancy until just prior to parturition, at which time fetal hormones in late gestation cause endocrine changes in the pregnant dam. This is observed as a large drop in progesterone levels in maternal blood serum, and indicates that parturition will typically occur within 2 to 5 days (Hess et al. 1983; Hodges et al. 1999; Dehnhard et al. 2003; Glaeser et al. 2012).

Cortisol and corticosterone are the two main circulating glucocorticoids which are commonly assessed to evaluate the stress response in Asian elephants (Kajaysri \& Nokkaew 2014). Cortisol has been shown to remain low throughout gestation until just prior to parturition. A cortisol spike is typical observed between 8 to 11 days prior to parturition and another is observed when active labor begins (Meyer et al. 2004). It is thought that the maternal cortisol surge is likely part of a cascade of endocrine events initiated by fetal hormones.

The endocrine profiles of gestating elephants, both for P4 and cortisol, have been previously described (Hess et al. 1983; Hodges et al. 1983; Olsen et al. 1994; 
Brown \& Lehnhardt 1995; Meyer et al. 2004). In this chapter, my objective was to determine whether our sample of five gestating elephants followed the expected patterns of a decrease in P4 and an increase in cortisol in the days prior to parturition. In Chapters 3 and 4, I will address the role of endocrine profiles as they relate to changes in behavior prior to parturition.

\section{Methods}

\section{Subjects and Study Sites}

We obtained endocrine profiles from regularly scheduled blood draws in five Asian elephant pregnancies at the Oregon and Taronga Zoos. At the Oregon Zoo, we observed the same elephant during her nulliparous and primiparous pregnancies. At the Taronga Zoo, we observed three elephants during their nulliparous pregnancies. (See Table 1.2, Chapter 1).

\section{Data Collection}

Endocrine monitoring occurred throughout gestation, with the rate of blood draws increasing in late gestation. The schedule for blood draws for each elephant was set by the veterinary staff and keepers at each institution (Table 2.1). Blood draws typically occurred in the morning hours at both institutions. If a second blood draw was required in the same day, it was taken in the afternoon. A drop in P4 of approximately $50 \%$ or greater from one sample to the next, with a concentration near or below 100 $\mathrm{pg} / \mathrm{ml}$, typically resulted in a scheduled increase in monitoring. 


\section{Analysis}

Hormone Analysis

All elephants were conditioned for routine blood draws from an ear vein (Taronga Zoo) or from an ear or leg vein (Oregon Zoo). The Oregon Zoo analyzed progesterone in blood serum using a solid-phase progesterone radioimmunoassay (Coat-a-Count progesterone; Siemens Diagnostic Solutions Corporation, Los Angeles, CA, USA). The antibody has a cross-reactivity of $9 \%$ to $5 \alpha$-DHP. Assay sensitivity was 50 $\mathrm{pg} / \mathrm{ml}$. This radioimmunoassay (RIA) has been successful in tracking progestogens, including P4, in Asian elephants (Brown et al. 1999). The radioimmunoassay (RIA) protocols used by the Oregon Zoo have been previously described (Glaeser et al. 2012). All P4 assays were completed in the Oregon Zoo Endocrinology Lab.

The Taronga Zoo sent their blood samples either to the College of Veterinary Science, University of Sydney or to the Wildlife Reproductive Centre Taronga Western Plains Zoo for enzyme-immunoassay (EIA). Progesterone was quantified using a monoclonal progesterone CL425 antibody previously validated for elephants (See Brown et al. 2004a; Lueders et al. 2010). The antibody has the following cross-reactivities: progesterone $100 \%$, 5 $\alpha$-pregnen-3, 20 dione $55 \%$ and $<0.1 \%$ pregnanediol, androstenedione, and corticosterone. Assay sensitivity was $0.05 \mathrm{ng} /$ well.

For cortisol analysis, the Oregon Zoo sent samples to the Oregon Health and Sciences University (OHSU) Endocrinology Lab. Blood serum cortisol was analyzed using 
the Immulite Assay in 2008 and the Roche Assay in 2012. Assay sensitivity and crossreactivities were not available at the time of publishing.

For the Taronga Zoo, cortisol was analyzed using a cortisol R4866 antibody previously validated for elephant serum (See Fanson et al. 2014). The antibody has the following cross-reactivities: cortisol $100 \%$, prednisolone $9.9 \%$, prednisone $6.3 \%$, cortisone $5 \%$ and $<1 \%$ corticosterone, desoxycorticosterone, 21-desoxycortisone, testosterone, androstenedione, androsterone, and 11-desoxycortisol. Assay sensitivity was $0.08 \mathrm{ng} / \mathrm{ml}$.

\section{Statistical Analysis}

The endocrine data were partitioned into three time periods based on the observed P4 drop in each elephant (Figure 2.1). The week before the P4 drop was selected as the Pre-period, and the days after the P4 drop were selected as the Postperiod. Note that the Post-period included data from one day prior to the recorded P4 drop. As blood draws were typically drawn each morning, a measured drop in P4 may have occurred at any time in the previous 24 hours. By including the day prior to the measured P4 drop in the Post period, I could ensure that any behaviors associated with the actual drop in P4 were captured in this period. In addition to the Pre- and Postperiods surrounding the P4 drop, a Baseline-period was defined as the three week time period prior to the Pre-period. I anticipated that the most behavioral change would occur just prior to and just after the P4 drop, as well as just prior to the final cortisol spike. 
There was considerable inter-individual variation in P4 and cortisol profiles, and as I was interested in the patterns of P4 and cortisol change, not the actual concentrations, P4 and cortisol were recoded as individual percentages. For each elephant, the greatest value for each profile became $100 \%$. All other values then became a percentage of the greatest value. While this did not remove inter-individual variation, it acted to trim extreme values while retaining the relative relationship of data points within an individual elephant. This allowed us to utilize patterns of P4 and cortisol change among the elephants, without having to account for extreme variation due to intrinsic individual differences or differing assay sensitivities between institutions.

An analysis of variance (ANOVA) was used to compare P4 and cortisol profiles among the three time periods across all elephants. In cases where a significant difference was reported, post-hoc Tukey tests were used to determine which time periods differed significantly. All analyses were performed in SPSS version 22.0.

\section{Results}

The final number of days included in the analysis, as well the number of days in each time partition, are given in Table 2.2.

\section{Endocrine Profiles}

The progesterone profiles for each elephant showed an expected drop in P4 in the days prior to parturition (Figure 2.2, a-e). The P4 drop occurred between 4 and 5 days prior to parturition in four of the elephants. However, in two of these cases (Figure $2.2 \mathrm{~b}$ and $2.2 \mathrm{c}$ ) the P4 drop was observed, but then P4 levels subsequently increased. For 
Rose-Tu in 2012 (Figure 2.2b), the observed drop to $59 \mathrm{pg} / \mathrm{ml}$ on day 643 of gestation was followed by a nearly 2 -fold increase to $115 \mathrm{pg} / \mathrm{ml}$ by day 647 , the day of parturition. For Pak Boon (Figure 2.2c), the observed drop to $30 \mathrm{pg} / \mathrm{ml}$ on day 654 of gestation was followed by a 12 -fold increase to $360 \mathrm{pg} / \mathrm{ml}$ the following day. Another drop was observed 2 days later, on gestation day 657, to $50 \mathrm{pg} / \mathrm{ml}$, and the level remained low until parturition on day 659. In one pregnancy (Porntip, Figure $2.2 \mathrm{~d}$ ), a drop was observed 10 days prior to parturition. The concentration decreased near the expected $100 \mathrm{pg} / \mathrm{ml}$, but due to complications with the pregnancy additional blood draws did not occur until the calf was born. Figure 2.3 shows all five elephants in the same chart, highlighting the temporal variation in P4 drop.

The cortisol profiles show an increase in serum cortisol levels in late gestation with high variability in both the concentration of cortisol and the timing of the surge (Figure 2.4). A spike was observed in one elephant as early as 3 weeks prior to parturition (Pak Boon).

To address inter-individual variation in endocrine levels, all statistical analyses used the endocrine data recoded as individual P4 and cortisol percentages. Individual percentages of P4 and cortisol are given in Figures 2.5 and 2.6, respectively.

An ANOVA compared P4 and cortisol levels among Baseline, Pre and Post time periods. There was significant variation in P4 levels $(F(2,152)=68.15, p<0.01)$ and cortisol levels $((F(2,152)=10.46, p<0.01)$ among the three time periods. A post-hoc 
Tukey HSD test indicates that the Post time period had significantly lower P4 levels and significantly higher cortisol levels than both the Baseline and Pre periods $(p<0.05)$.

\section{Discussion}

I observed generally expected endocrine patterns in all five pregnancies, with a drop in progesterone in all five pregnancies. Levels of P4 were significantly lower in the time period after this drop (the Post period) than in both the Baseline and Pre periods. I also found significantly higher levels of cortisol in the Post time period after the P4 drop than in both the Baseline and Pre periods.

Progesterone monitoring tracks luteal activity in late gestation, with a drop in progesterone levels indicating luteolysis has occurred and parturition is imminent (Senger 2003; Szdzuy et al. 2006). In the Asian elephant, the mechanisms for these changes in circulating maternal hormones is still under study, though Meyer et al. (2004) suggest they may be similar to other mammalian species. Fetal cortisol has been shown to start a cascade of events that initiates parturition in other mammalian species, including sheep (Rees et al. 1975, Challis et al. 2000), pigs (Kattesh et al. 1997), and the wallaby (Ingram et al. 1999). In other mammalian species, activation of the fetal hypothalamic-pituitary-adrenal (HPA) axis and increased fetal cortisol may induce two primary outcomes via normal endocrine actions: conversion of maternal progesterone to estradiol, and driving maternal hormones to regress the corpus luteum (Senger 2003). If validated in the Asian elephant, these changes may account for the drastic drop 
observed in circulating maternal progesterone that initiates the beginning stages of parturition.

While I found that in general P4 followed expected patterns of a drop in late gestation (Meyer et al. 2004), I also noted that three of the five elephants showed differences worth noting. For example, one elephant (Porntip) showed a drop of greater than $50 \%$, with a concentration near $100 \mathrm{pg} / \mathrm{ml}$. This was determined to be the day of the drop in P4. The dam showed signs of hard labor, yet a calf was not produced for another 10 days, well outside the expected 2-5 days. In Taronga birth protocols, cases have been reported with up to 14-days between an observed P4 drop and delivery of a live calf.

I also report that in two of five pregnancies there was an increase in P4 levels after the observed P4 drop. These increases were only observed in the Oregon Zoo female (Rose Tu). These intra-individual variations can make real-time interpretation of the P4 drop challenging (Sharon Glaeser pers. comm. 2016). Thus, relying solely on P4 drop as a predictor can be problematic. In general, cortisol followed expected patterns of increased levels in late gestation (Meyer et al. 2004). Intra-individual cortisol levels were particularly variable, however, with one elephant (Pak Boon) showing a spike three weeks prior to parturition. This diverges from previous studies in which the peak was within 2 weeks of parturition (Meyer et al. 2004).

Considerable inter- and intra-individual variation in P4 and cortisol levels is not unexpected (Meyer et al. 2004). P4 levels can vary by sex of the fetus, with P4 levels 
being higher when carrying a male fetus than a female fetus (Meyer et al. 2004). Cortisol is a metabolic hormone produced by the adrenal glands that helps to mediate responses to real or perceived stressful stimuli. While it does surge due to pregnancy and impending parturition, it cannot be isolated from other stressful events that may result in cortisol fluctuations.

Observing these challenges with endocrine profiles in even this small sample set highlights the need for additional markers, such as behaviors, to support late gestation management. 


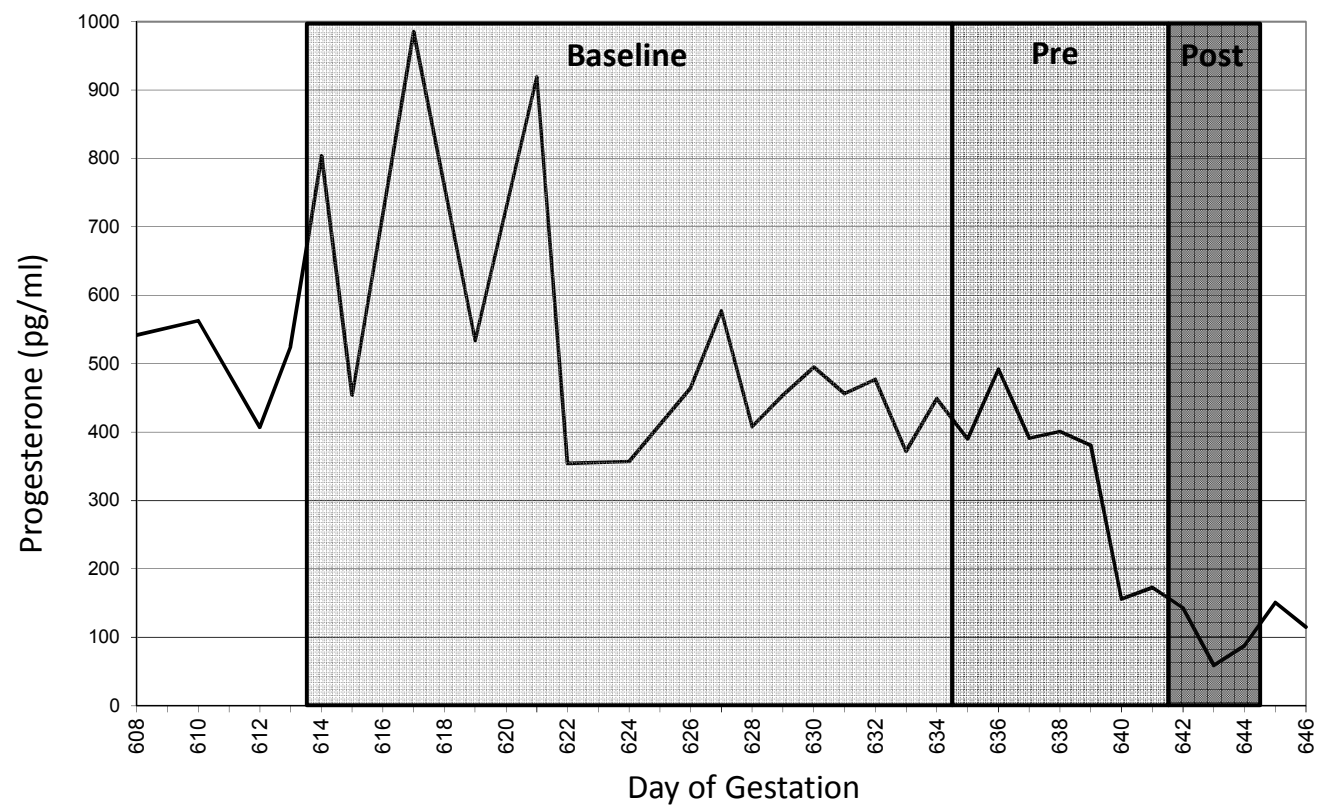

Figure 2.1. Progesterone data partitioned into Baseline, Pre and Post time periods for a representative animal from the study. 
(a)

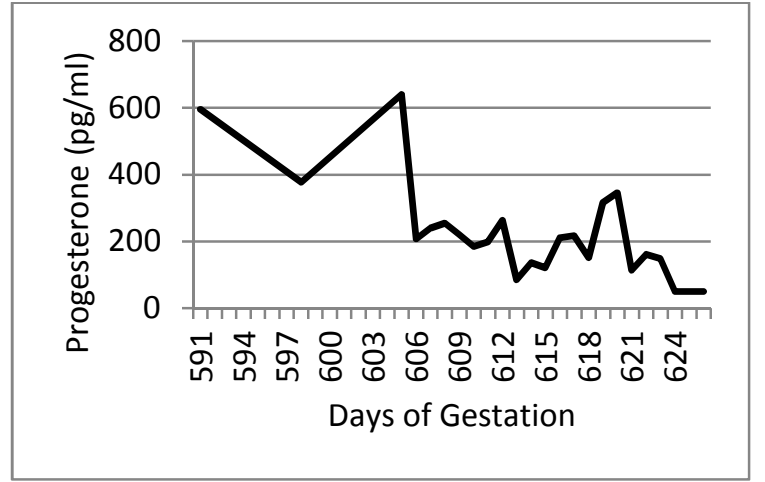

(c)

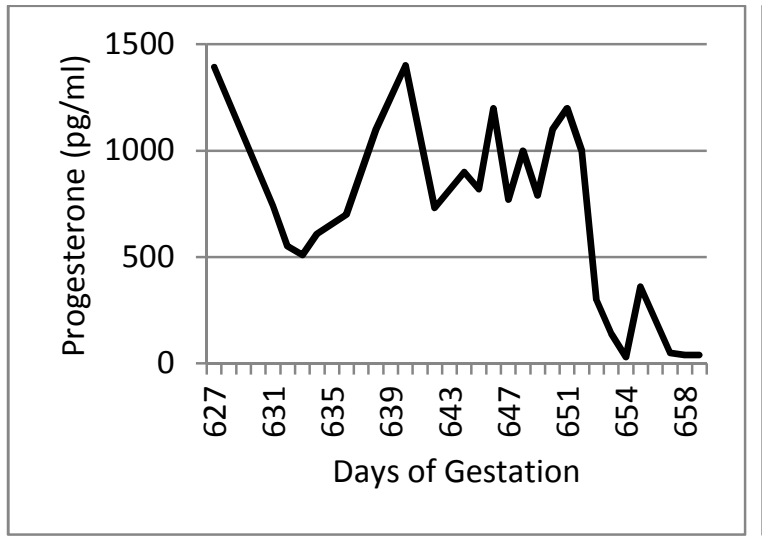

(e)

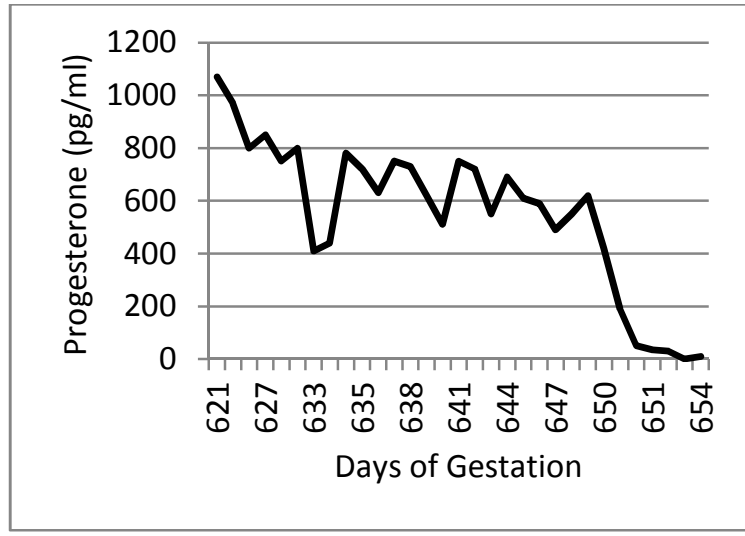

(b)

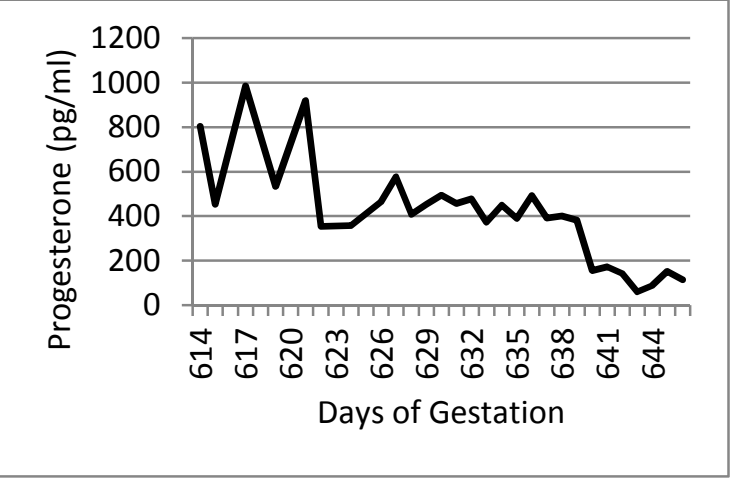

(d)

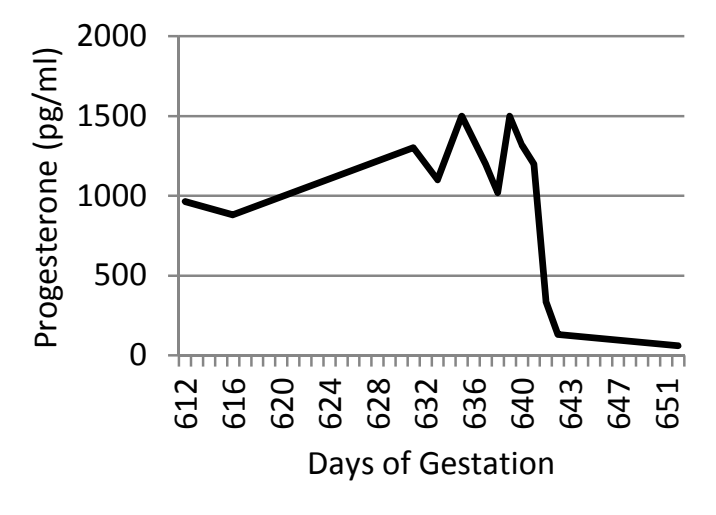

Figure 2.2. Individual profiles show a decrease in P4 levels in the days prior to parturition for (a) RoseTu 2008; (b) Rose-Tu 2012; (c) Pak Boon; (d) Porntip; (e) Thong Dee 


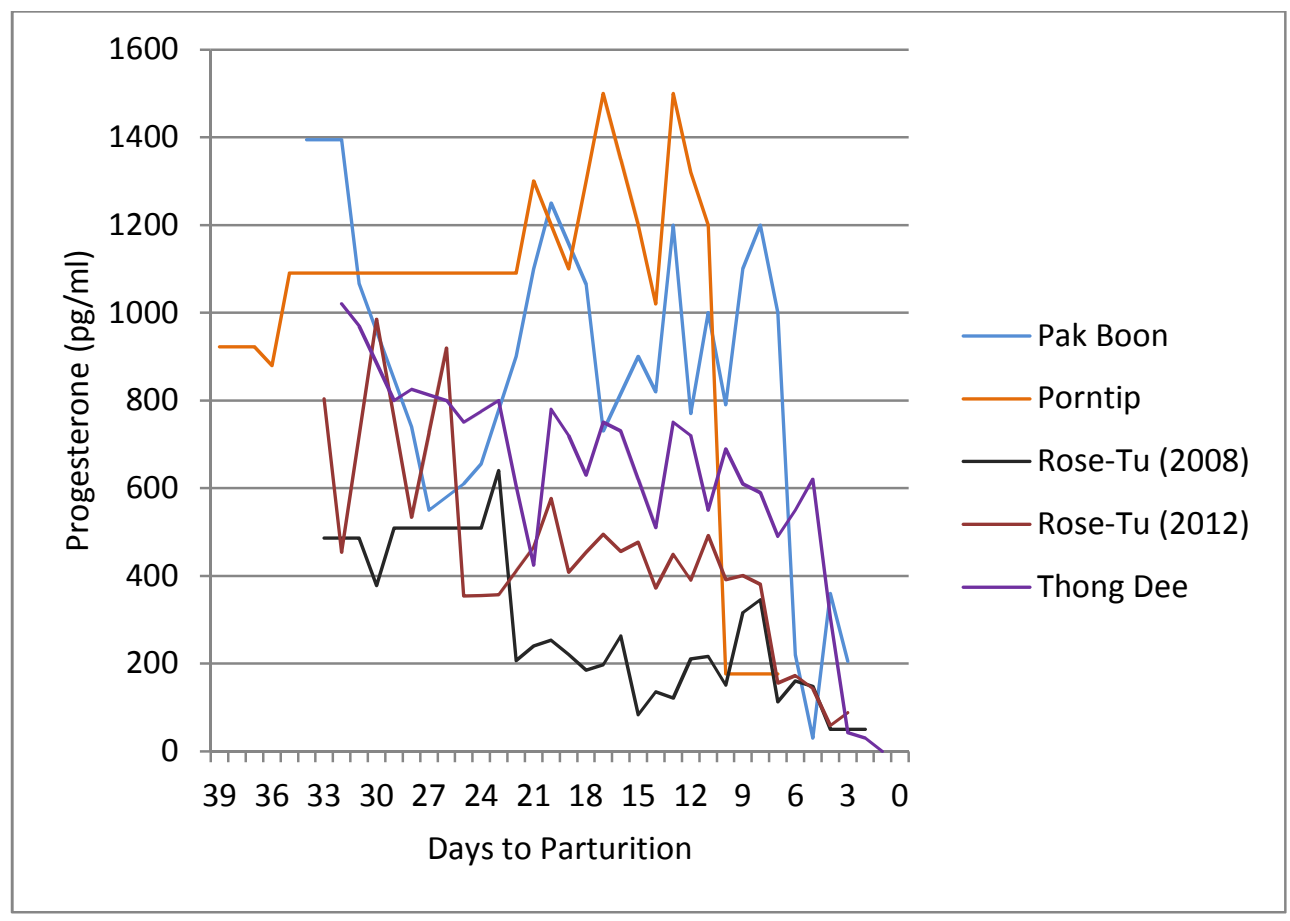

Figure 2.3. Late gestation progesterone profiles show a decrease in P4 concentration in the days prior to parturition for all elephants

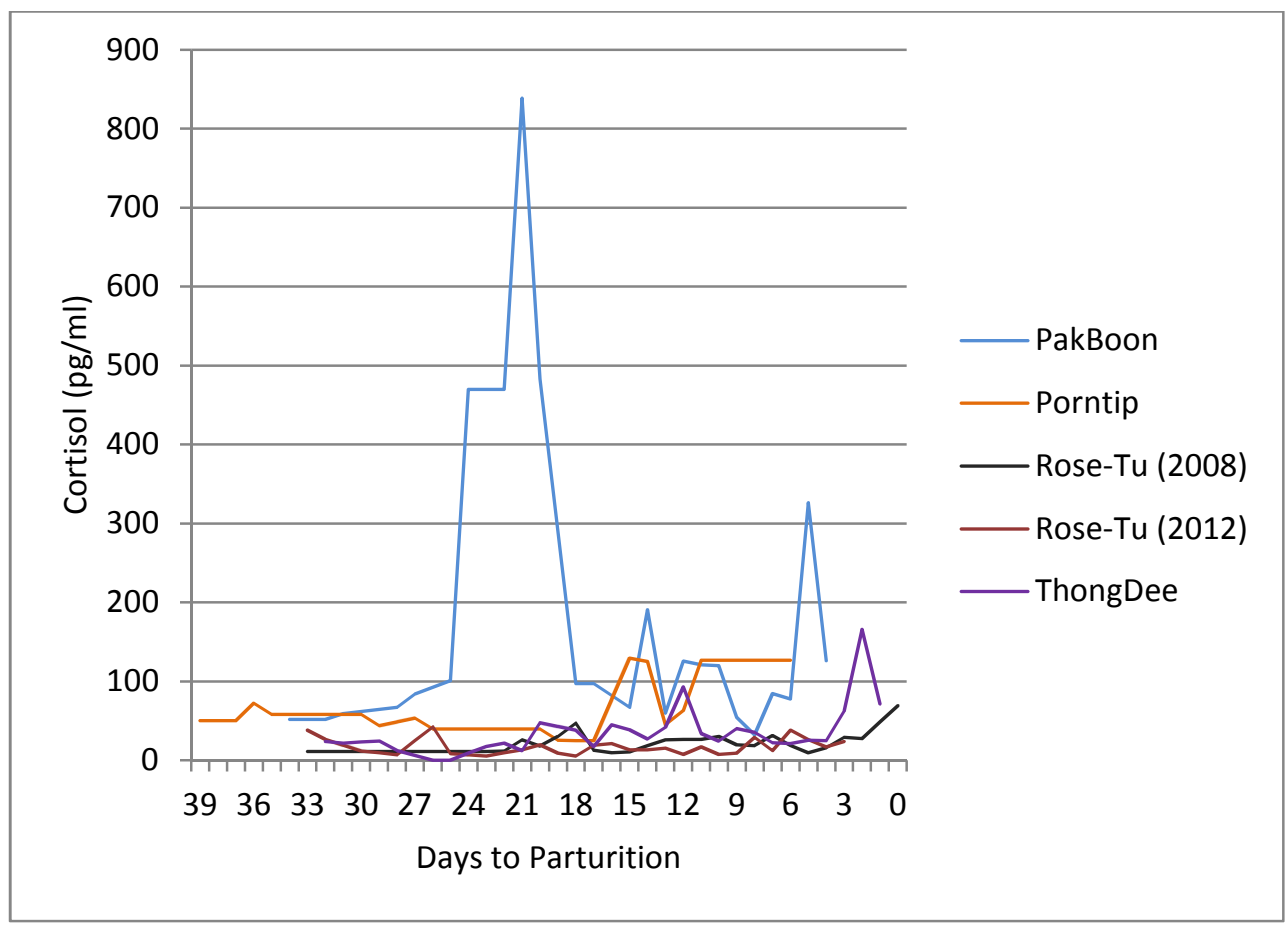

Figure 2.4. Late Gestation Cortisol Profiles 


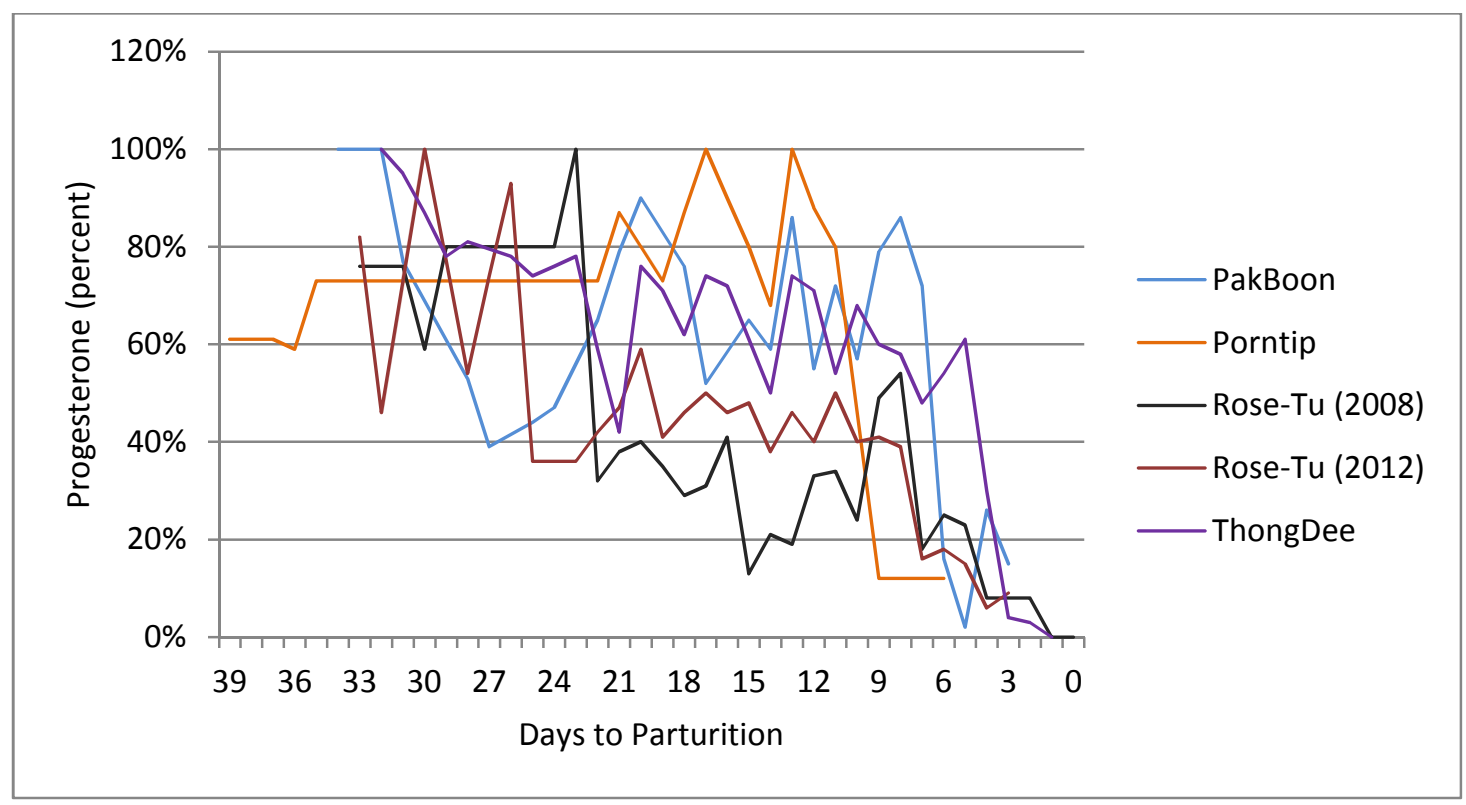

Figure 2.5. Late Gestation Progesterone Profiles Reported as a Percentage of Each Elephant's Maximum

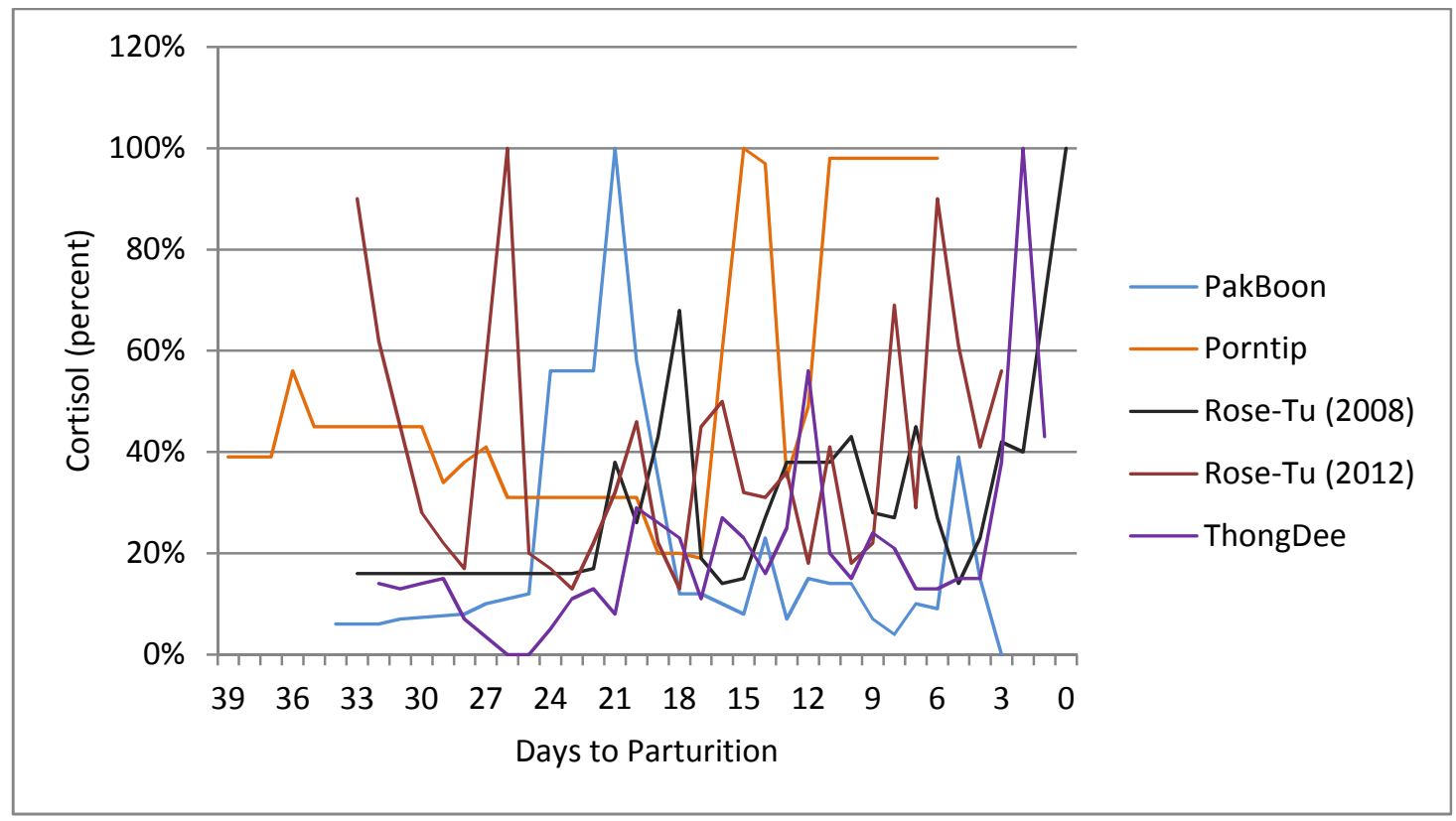

Figure 2.6. Late Gestation Cortisol Profiles Reported as a Percentage of Each Elephant's Maximum 
Table 2.1. Schedule of Blood Draws for Duration of Gestation, By Elephant

\begin{tabular}{lcccccc}
\hline Elephant & \multicolumn{3}{c}{ Blood Collection Schedule (By Day of Gestation, Unless Otherwise Indicated) } \\
\cline { 2 - 7 } & $\begin{array}{c}\text { Every } \\
\text { other } \\
\text { week }\end{array}$ & Weekly & $\begin{array}{c}\text { Twice } \\
\text { Weekly }\end{array}$ & $\begin{array}{c}\text { Every } \\
\text { Other } \\
\text { Day }\end{array}$ & Daily & Twice Daily \\
\hline RoseTu 2008 & & Up to 604 & & & $605-$ Delivery & \\
\hline RoseTu 2012 & & Up to 609 & $610-626$ & & 626 to P4 Drop & P4 Drop to Delivery \\
\hline Pak Boon & Up to 589 & $590-620$ & $621-634$ & $635-642$ & 643 to P4 Drop & P4 drop until Birth \\
\hline Porntip & Up to 589 & & $590-620$ & $621-634$ & 635 to P4 Drop & P4 Drop to Delivery \\
\hline Thong Dee & Up to 629 & & & $630-639$ & 640 to P4 Drop & P4 Drop to Delivery \\
\hline
\end{tabular}

Table 2.2. Day Count of Records Used in Preparturition Analysis

\begin{tabular}{lcccc}
\hline & $\begin{array}{c}\text { Days of Data } \\
\text { Utilized for } \\
\text { Preparturition } \\
\text { Analysis }\end{array}$ & $\begin{array}{c}\text { Days of } \\
\text { Baseline Data }\end{array}$ & $\begin{array}{c}\text { Days of Pre } \\
\text { Data }\end{array}$ & $\begin{array}{c}\text { Days of } \\
\text { Post Data }\end{array}$ \\
\hline PornTip & 33 & 21 & 7 & 5 \\
Thong Dee & 31 & 20 & 7 & 4 \\
PakBoon & 26 & 15 & 7 & 4 \\
RoseTu (2008) & 34 & 21 & 7 & 6 \\
RoseTu (2012) & 31 & 21 & 7 & 3 \\
\hline
\end{tabular}




\section{Chapter 3: Preparturition Behaviors as Predictive Tools in Late Gestation}

\section{Introduction}

Predicting elephant parturition using hormone data and ultrasound technology has been described in detail and incorporated into birth protocols for decades (Hess et al. 1983; Hodges et al. 1999; Dehnhard et al. 2003; Schmidt 1982). Routine endocrine sampling is used by many Association of Zoos and Aquariums (AZA) institutions to monitor estrous cycles, to detect or confirm pregnancy, and to predict parturition. Based on responses to a 2001-2002 Elephant Taxon Advisory Group (TAG)/Species Survival Plan (SSP) reproductive survey, approximately $49 \%$ of Asian and $62 \%$ of African elephants in North America undergo weekly endocrine/hormonal monitoring for estrous cyclicity (Brown et al. 2004b). This means a number of institutions either do not employ endocrine monitoring, or do not do so regularly. These institutions often lack access to endocrine labs or the funds to perform regular endocrine monitoring.

Additional tools are needed to support those institutions that cannot employ routine endocrine monitoring. Behaviors associated with parturition are anecdotally mentioned in elephant birth protocols, yet there is a lack of examination of behavioral data to predict parturition. If validated, behavioral predictors could be useful not only to organizations that do not have regular endocrine assessments, but also to organizations that do perform endocrine analyses, especially when data are missing, analysis results are unreliable, or endocrine sampling timelines need support. As previously discussed, 
endocrine assessments can be difficult to interpret in real time, especially due to individual fluctuations or variation (see Chapter 2).

My objective was to investigate whether behaviors previously considered anecdotal by zoo keepers and veterinary staff could be predictive of impending parturition in the Asian elephant.

\section{Methods}

\section{Subjects and Study Sites}

We observed late gestation behavior in five Asian elephant pregnancies at the Oregon and Taronga Zoos. At the Oregon Zoo, we observed the same elephant during her nulliparous and a subsequent primiparous pregnancy. At the Taronga Zoo, we observed three elephants during their nulliparous pregnancies. (See Table 1.2, Chapter 1).

\section{Data Collection}

The preparturition behaviors selected for the ethogram were based on anecdotal evidence in birth protocols, conversations with zoo keepers and staff, and literature searches (Szdzuy et al. 2006; Rees 2009; Horback et al. 2014). The selected behaviors and their descriptions are provided in Table 3.1. Behavior observations of each elephant began by approximately day 600 of gestation and continued until the calf was delivered. There were four scheduled, 30-minute observations per day, seven days per week. A 30minute observation was scheduled to begin and end in each of the following 2-hour 
windows: 9:00-10:59; 11:00-12:59; 13:00-14:59; and 15:00-17:00. The "all-occurrences" sampling method was used for all preparturition behaviors (Martin \& Bateson, 2007).

\section{Analysis}

Some variables were combined for analysis (Table 3.2). In two cases, this was done to account for inconsistencies in data collection between institutions. For example, at Taronga Zoo, some observers recorded when a stance change occurred without indicating the type of stance change. In order to keep as much data as possible, these data were aggregated into a more generic "stance change" variable. Similarly, Taronga Zoo recorded rocking and swaying as a single, combined variable, while the Oregon Zoo recorded these as two separate variables. While aggregation resulted in the loss of detail in the Oregon zoo data set, this was preferred over the loss of data points in the full data set.

I partitioned the data into the three time periods described in Chapter 2, based on the observed P4 drop in each elephant: Baseline, Pre, (the week period preceding the P4 drop), and Post (the period after the P4 drop).

I initially analyzed two data sub-sets: binary data and non-zero data. In the binary data set, behaviors were recorded as present or absent for each day (1/0), but the actual number of occurrences was not analyzed. In the non-zero data set, all absent behaviors (i.e. zeros) were removed from the data set in order to determine the actual number of times a behavior occurred. This provided some flexibility in the statistical analyses that were used, and allowed us to analyze whether presence or absence of a 
behavior was as important as the frequency of a behavior. Following the sub-set analyses, I analyzed the full data set. Significant results or trends from the sub-set analyses helped to inform some of variables selected in modeling the full data set. All analyses were performed in SPSS version 22.0.

\section{Binary Data Set Analysis}

A likelihood ratio was determined using the binary data set. I compared the presence or absence of each preparturition behavior as a function of the timepartitioned data (baseline, pre and post periods).

\section{Non-Zero Data Set Analysis}

Initially, an ANOVA was used to compare the means for each trunk-check behavior for the Baseline, Pre and Post time periods. Data were examined for normality assumptions using the Shapiro-Wilk test. In cases where a significant result was found, a post-hoc Tukey test was used to determine which time periods differed significantly.

Due to concerns regarding normality assumptions, an additional non-parametric test was performed. A Kruskal-Wallis test (a rank-based non-parametric test similar to an ANOVA) does not rely on normality or equal variances among groups. The KruskalWallis test was used with the non-zero data set to compare the means for each preparturition behavior among the Baseline, Pre and Post time periods. In cases where a significant result was reported, mean ranks were compared and interpreted. 


\section{Full Data Set Analysis}

I used principal component analysis and step-wise reduction of generalized linear mixed models to evaluate preparturition behaviors. Prior to analysis, all variables were examined for normality and variance assumptions.

A principal component analysis (PCA) is a dimension reduction procedure that is useful in cases where variables may be correlated. It transforms individual variables into aggregate variables, or multivariable components, that are not correlated. This procedure typically results in a fewer number of components than the original number of variables, thus it "reduces dimension". I retained components for further modeling analyses with an Eigen value greater than 1.0, or falling above the elbow on the PCA scree plot. The PCA components were renamed by their highest loading variables.

Generalized Linear Mixed Models (GLMMs) with an identity link function and a normal probability distribution were used to further evaluate the data. A GLMM was used to determine what fixed effects and covariates, if any, accounted for the variance within a response, or dependent, variable. I first ran GLMM to examine each PCA component as a response variable. In each model, the time partition (Baseline, Pre and Post) was entered as a fixed effect categorical variable. Both P4 and cortisol percentage profiles were entered as continuous covariates. Elephant identity and institution were entered as random effects, to account for excess variability in the response variable.

Subsequently, GLMM were used to examine individual preparturition behaviors as the response variable. Behaviors were selected that showed significance or trends in 
the binary or non-zero data set analyses. Fixed effects, covariates, and random effects remained as described above.

\section{Results}

\section{Binary Data Set}

I examined each preparturition behavior as a function of the time-partitioned data (Baseline, Pre and Post periods), using a likelihood ratio test. A significant result indicates there was a difference in the presence or absence of the behavior among the three time periods.

Among all the variables, only the Dust Environment behavior showed a significant difference in occurrence of the behavior among the three partitioned time periods. However, closer examination of the data indicated that the overall occurrence of the behavior was so infrequent (less than 10 occurrences) that further analysis was not warranted.

Along with the Dust Environment behavior, additional variables were identified with very low frequency of occurrence $(n<10)$ : Kicking Belly, Tail Tuck, and Strike Behaviors. These variables were removed from further analyses.

\section{Non-Zero Data Set}

Most variables in the non-zero data set did not meet normality assumptions as required for an ANOVA. Although the data were log transformed, the majority of the variables did not pass the Shapiro-Wilk test for normality. Exceptions were the (log) Head Down and (log) Rocking/Swaying variables, which did pass the Shapiro-Wilk test. 
However, an examination of the histograms and Q-Q plots of all the transformed variables showed that many of the variables appeared normal. Because very small sample sizes can affect the outcome of the Shapiro-Wilk test, I ran both an ANOVA (Table 3.4) and a non-parametric Kruskal-Wallis test (Table 3.6), on the non-zero data set.

The ANOVA results indicated a significant difference in the amount of time that elephants spent Walking Backward, in the three time periods $(F(2)=3.723, p=0.033)$ (Table 3.4). Post-hoc Tukey HSD testing could not resolve these differences, but showed a trend with higher levels of Walking Backward in the Post time period than the Baseline period $(p=0.059)$. There was no difference found between the Baseline vs Pre periods or the Pre vs Post time periods (Table 3.5).

The non-parametric Kruskal-Wallis test also indicated a significant difference in the amount of time that elephants spent walking backward, among the three time periods $(H(2)=6.289, p<0.05)$, with a mean rank of 18.7 for baseline, 27.0 for pre, and 33.7 for the post period. While a multiple comparisons test could not resolve the differences among groups, there was a trend in increased Walking Backward behavior as the elephants move from Baseline to Pre, and Pre to Post time periods.

\section{Full Data Set}

A PCA analysis identified three multi-variable components (Table 3.7). Component 1 accounted for $23 \%$ of overall variance, Component 2 accounted for an additional $14 \%$ of variance, and Component 3 accounted for $13 \%$ of variance. In total, 
the three components accounted for $50 \%$ of the variance in the data. The variables with the highest loadings, and therefore accounting for the greatest variance, in Component 1 were Stance Change, Head Down, Rubbing, Dusting Belly/Flanks and Lying Down. This component was renamed the "Body Changes" variable, after the highest loading variables which were the drivers in accounting for the greatest variance in the component. In Component 2, renamed the "Dusting" variable, the variables with the highest loadings were Dusting Overhead and Stretching. In Component 3, renamed "Movement", the variables with the highest loadings were Walking Backwards and Rocking/Swaying.

I used GLMM to incorporate time partitions and endocrine profiles into our analyses. A separate GLMM was performed using each PCA component as a target to determine if time partitions and endocrine profiles accounted for the variance within each component. The model using Body Change (Component 1 ) as a function of time partition, P4 and cortisol was not significant (Table 3.8). To determine if there was a hidden effect among them, additional GLMM models were run with each fixed effect tested separately. None of the fixed effects of time partition, P4 or cortisol were significant (Table 3.9). As with the Body Change component model, no significant fixed effects were found in additional GLMM models for Dusting (Component 2) and Movement (Component 3). In all models, random effects (Elephant ID and Institution) were significant, meaning there were significant differences in the PCA component values among elephants and between institutions. Thus, the variation among individuals 
and between institutions is high. The models accounted for this variation by holding these factors constant.

In addition to the PCA Component models, I ran GLMM models using individual behaviors that had shown significance or trends in the likelihood ratio, ANOVA and/or Kruskal-Wallis analyses. I found a significant model for Walking Backwards as a function of the P4 and cortisol levels. A stepwise reduction approach resulted in a best-fit model with only the P4 levels as a fixed factor (Table 3.10). According to the model, as P4 levels decrease, Walking Backwards behavior increases. The random effects (Elephant ID and Institution) were significant, meaning there were significant differences in the Walking Backwards values among elephants and between institutions. Thus, the variation among individuals and between institutions is high. The model accounted for this variation by holding these factors constant.

\section{Discussion}

I investigated twelve preparturition behaviors to evaluate their role in predicting parturition. I report an increase in Walking Backward behavior across the three time periods (Baseline, Pre and Post) as parturition approached. This result was also supported in a GLMM model in which Walking Backwards varied with the time partitions. A significant effect was seen for P4 in the Walking Backward behavior. As P4 levels decreased, there was an increase in walking backwards.

I am not the first to report Walking Backwards as a behavior observed during preparturition. Leuthold \& Leuthold (1975) first reported observing this behavior in a 
wild African elephant. The behavior caught their attention as being "unusual", as it differed from the other elephants in the group. They were unaware that the observed elephant was in labor until she produced a calf a short time later, surrounded by female herd-mates.

Despite this early observation of Walking Backwards, there is little information on the role of this behavior in the preparturient period, but it may play a role in reducing or changing stress on the joints, especially the hind limbs, or the pelvic rim. As noted in the Taronga birth protocols, in late gestation the dams may attempt to climb up substrates or other surfaces with their hind legs to reduce the calf pressure on the pelvic rim while also allowing the calf to optimize its position for birth. It is feasible that Walking Backwards is performed in a dam's attempt to find higher ground for the hind limbs.

I tested models for the significant increase in Walking Backwards across time periods, and the changes in this behavior were associated with changes in P4. However, I recognize that these models may not be practical predictive tools from a management standpoint, as I cannot predict the number of days to parturition based on a specific change in the Walking Backward behavior. However, keepers, veterinary staff, and other observers that are familiar with the regular behavioral repertoire of a pregnant female should pay close attention to the Walking Backward behavior. Keeping track of this behavior, especially in conjunction with P4 tracking, can help staff provide a window of expected parturition. I recommend that institutions provide space for the pregnant dam 
to walk backwards, both in the daytime and at night, as well as provide elevated substrate in order for the dam to elevate her hind quarters.

In our study, no other observed preparturition behaviors showed significant changes over the Baseline, Pre and Post time periods, nor showed variations with P4 or cortisol profiles. The preparturition behaviors did show high variation between individuals and institutions. 
Table 3.1. Ethogram Variables for Preparturition Behaviors

\begin{tabular}{|c|c|}
\hline Behavior & Description \\
\hline Lying Down & Side, stomach or back is in contact with the substrate \\
\hline Kicking Belly & Kicks upwards at or makes contact with belly with any foot \\
\hline Walking Backwards & Walks backward for 3 or more seconds \\
\hline Stance Change & Includes the following specific stance-change types: \\
\hline Squat & Hind legs bent, rump is lower than head \\
\hline Sit & Rump is touching the ground and front legs used as support \\
\hline Kneel & $\begin{array}{l}\text { Hind knee(s) touching ground or front wrist(s) curled and touching the } \\
\text { ground }\end{array}$ \\
\hline Legs Crossed & $\begin{array}{l}\text { Hind legs crossed; one leg/foot hooked over other; one leg not fully weight- } \\
\text { bearing }\end{array}$ \\
\hline Legs Splayed & Feet are wider than hips (Not used while urinating/defecating) \\
\hline Head Down & $\begin{array}{l}\text { Head dropped or hind legs walked up ramp/surface so head is lower than } \\
\text { rump }\end{array}$ \\
\hline Rubbing & $\begin{array}{l}\text { A bout of rubbing against walls, objects, other elephants (Does not include } \\
\text { manipulating browse to scratch or rubbing/scratching feet) }\end{array}$ \\
\hline Stretching & $\begin{array}{l}\text { A bout of stretching lasting more than } 2 \text { minutes: Walks front legs out from } \\
\text { under body and leans forward so back legs are no longer under body; one } \\
\text { or more limbs stretched out and away from the body; any stretch using a } \\
\text { prop or wall; (Does not include typical leg stretching, in which a leg is } \\
\text { extended forward with the heel placed on the ground) }\end{array}$ \\
\hline Rocking & $\begin{array}{l}\text { All four feet are on the ground, moves body front to back without } \\
\text { stretching }\end{array}$ \\
\hline Swaying & All four feet are on the ground, moves body side to side \\
\hline Tail Beating & $\begin{array}{l}\text { A bout of tail beating: Tail is moved in up and down motion } 2 \text { or more times } \\
\text { (tail beating); tail is moved in side-to-side motion } 2 \text { or more times (tail } \\
\text { flagging); or tail is lifted erect, dropped between legs, dragged across } \\
\text { clitoris, then flicked or waved like a flag (tail flicking) (Does not include } \\
\text { normal tail "swishing") }\end{array}$ \\
\hline Dusting Behaviors & Dusting behaviors include the following specific dusting-types: \\
\hline Dusting Belly/Flanks & $\begin{array}{l}\text { Uses trunk to throw sand, dirt, wood chips/shavings, mud or water towards } \\
\text { self in direction of belly/flanks }\end{array}$ \\
\hline Dusting Overhead & $\begin{array}{l}\text { Uses trunk to throw sand, dirt, wood chips/shavings, mud or water towards } \\
\text { self, directed overhead. }\end{array}$ \\
\hline Dusting Environment & $\begin{array}{l}\text { Uses trunk to throw sand, dirt, wood chips/shavings, mud or water not } \\
\text { directed towards self }\end{array}$ \\
\hline Tail Tuck & Tucking the tail of another elephant under her front leg (armpit) \\
\hline Strike & $\begin{array}{l}\text { Strikes Include: Hitting another elephant with forehead or trunk; Kicking } \\
\text { another elephant with any foot }\end{array}$ \\
\hline
\end{tabular}


Table 3.2. Variable Aggregation for Analysis

\begin{tabular}{ll}
\hline Behavior & Description of Aggregation \\
\hline Stance Change & $\begin{array}{l}\text { All stance changes types were combined into a single "stance change" } \\
\text { variable }\end{array}$ \\
$\begin{array}{l}\text { Rocking/Swaying } \\
\text { Rusting Behaviors }\end{array}$ & $\begin{array}{l}\text { Dusting behaviors were examined in aggregate and in the three separate } \\
\text { dusting-type behaviors (Belly/Flanks, Overhead, Environment) }\end{array}$ \\
\hline
\end{tabular}

Table 3.3. Likelihood Ratio Test for Preparturition Behaviors as a function of Partitioned Time (Baseline, Pre and Post) $\left({ }^{*} p<0.05\right)$

\begin{tabular}{ll}
\hline Variable & Likelihood Ratio P-values \\
\hline Lying Down & 0.310 \\
Kicking Belly & 0.195 \\
Walking Backwards & 0.262 \\
Stance Change & 0.167 \\
Head Down & 0.618 \\
Rubbing & 0.712 \\
Stretching & 0.787 \\
Rocking/Swaying & 0.379 \\
Tail Beating & 0.428 \\
Dust Belly/Flanks & 0.708 \\
Dust Overhead & 0.360 \\
Dust Environment & $0.041^{*}$ \\
$\quad$ Dust-Aggregate & 0.515 \\
Tail Tuck & 0.151 \\
Strike & 0.784 \\
\hline
\end{tabular}


Table 3.4. ANOVA: Preparturition Behaviors in Baseline, Pre and Post Time Periods, With All Individuals Combined $(* p<0.05)$.

\begin{tabular}{llll}
\hline Variable & Df & F-Statistic & $\begin{array}{l}\text { ANOVA F-Statistic } \\
\text { P-values }\end{array}$ \\
\hline (Log)Lying Down & 2 & 0.406 & 0.673 \\
(Log)Walking Backwards & 2 & 3.723 & $0.033^{*}$ \\
(Log)Stance Change & 2 & 0.255 & 0.775 \\
(Log)Head Down & 2 & 0.095 & 0.911 \\
(Log)Rubbing & 2 & 0.672 & 0.514 \\
(Log)Stretching & 2 & 0.875 & 0.424 \\
(Log)Rocking/Swaying & 2 & 2.437 & 0.097 \\
(Log)Tail Beating & 2 & 0.269 & 0.767 \\
(Log)Dust Belly/Flanks & 2 & 1.047 & 0.354 \\
(Log)Dust Overhead & 2 & 0.417 & 0.661 \\
(Log)Dust-Aggregate & 2 & 0.135 & 0.874 \\
\hline
\end{tabular}

Table 3.5. Post-Hoc Comparisons Between Baseline, Pre \& Post periods, With All Individuals Combined

\begin{tabular}{llll}
\hline Variable & $\begin{array}{l}\text { Time Partition } \\
\text { Comparison }\end{array}$ & $\begin{array}{l}\text { Tukey HSD Mean } \\
\text { Difference }\end{array}$ & $\begin{array}{l}\text { Statistic P- } \\
\text { values }\end{array}$ \\
\hline (Log)Walking Backwards & Baseline vs Pre & -0.202 & 0.244 \\
& Pre vs Post & -0.195 & 0.588 \\
& Baseline vs Post & -0.397 & 0.059 \\
\hline
\end{tabular}

Table 3.6. Kruskal-Wallis Preparturition Behaviors as a Function of Partitioned Time (Baseline, Pre and Post) $(* p<0.05)$

\begin{tabular}{ll}
\hline Variable & $\begin{array}{l}\text { Kruskal-Wallis Test } \\
\text { Statistic P-value }\end{array}$ \\
\hline Lying Down & 0.505 \\
Walking Backwards & $0.043^{*}$ \\
Stance Change & 0.774 \\
Head Down & 0.991 \\
Rubbing & 0.453 \\
Stretching & 0.185 \\
Rocking/Swaying & 0.081 \\
Tail Beating & 0.571 \\
Dust Belly/Flanks & 0.328 \\
Dust Overhead & 0.528 \\
\multicolumn{1}{c}{ Dust-Aggregate } & 0.838 \\
\hline
\end{tabular}


Table 3.7. Variable Loadings by Component (highest loadings in bold).

\begin{tabular}{lccc}
\hline Variable & $\begin{array}{c}\text { Component 1 } \\
\text { (Body Change) }\end{array}$ & $\begin{array}{c}\text { Component 2 } \\
\text { (Dusting) }\end{array}$ & $\begin{array}{c}\text { Component 3 } \\
\text { (Movement) }\end{array}$ \\
\hline Lying Down & .578 & -.263 & .313 \\
Walking Backwards & -.096 & .007 & -.713 \\
Stance Change & .753 & .304 & .084 \\
Head Down & .660 & -.251 & .195 \\
Rubbing & .653 & -.019 & -.279 \\
Stretching & .026 & .664 & -.155 \\
Rocking/Swaying & -.235 & .199 & .680 \\
Tail Beating & -.098 & .349 & .214 \\
Dusting Belly/Flanks & .633 & .118 & -.121 \\
Dusting Overhead & .122 & .748 & .017 \\
\hline
\end{tabular}

Table 3.8. GLMM PCA Body Change Component with Data Partition, P4, and Cortisol as Fixed Effects

\begin{tabular}{lllll}
\hline Source & F-Statistic & df1 & df2 & Significance \\
\hline Corrected Model & 0.443 & 4 & 149 & 0.777 \\
Time Partition & 0.764 & 2 & 149 & 0.468 \\
P4 & 0.591 & 1 & 149 & 0.443 \\
Cortisol & 0.494 & 1 & 149 & 0.483 \\
\hline
\end{tabular}

Table 3.9. GLMM Models with Body Change Component and Each Fixed Effect

\begin{tabular}{|c|c|c|c|c|}
\hline Model & F-statistic & df1 & df2 & Significance \\
\hline Full Model: Body Change Time Partition + P4 + Cortisol & 0.443 & 4 & 149 & 0.777 \\
\hline Sub Model: Body Change Time Partition & 0.244 & 2 & 152 & 0.784 \\
\hline Sub Model: Body Change P4 + Cortisol & 0.122 & 2 & 151 & 0.885 \\
\hline Sub Model: Body Change P4 & 0.035 & 1 & 152 & 0.851 \\
\hline Sub Model: Body Change Cortisol & 0.246 & 1 & 152 & 0.621 \\
\hline
\end{tabular}

Table 3.10. GLMM Walking Backwards (Best Fit Model in Bold)

\begin{tabular}{lllll}
\hline Model & F-statistic & df1 & df2 & P-value \\
\hline Full Model: Walking Backwards Time Partition + P4 + Cortisol & 2.045 & 4 & 152 & 0.091 \\
Sub Model: Walking Backwards Time Partition & 2.942 & 2 & 152 & 0.056 \\
Sub Model: Walking Backwards P4 + Cortisol & 3.566 & 2 & 151 & $0.031^{*}$ \\
Sub Model: Walking Backwards P4 & 6.313 & $\mathbf{1}$ & $\mathbf{1 5 2}$ & $\mathbf{0 . 0 1 3 ^ { * }}$ \\
Sub Model: Walking Backwards Cortisol & 2.860 & 1 & 152 & 0.093 \\
\hline
\end{tabular}




\section{Chapter 4: Trunk-Check Behaviors as Predictive Tools in Late Gestation}

\section{Introduction}

Elephant trunks are multifaceted tools used to investigate surroundings, for eating and drinking, olfaction, and in the flehmen response for detecting pheromones (Rasmussen \& Schulte 1998; Blogg, 2012). In this chapter, I examine investigative behaviors using the trunk directed towards the pregnant dam. We refer to these behaviors as "trunk-checks", and they are performed by the pregnant dam in "self trunk-checks", and by others towards the pregnant dam in "herd-mate trunk-checks".

Behaviors associated with parturition are only anecdotally mentioned in elephant birth protocols (see Chapter 3). These include behaviors which may be associated with physical and physiological changes in the pregnant dam in late gestation. For example, one birth protocol lists pulling of teats as an anecdotal behavior in late gestation. This trunk-checking behavior may be the result of the onset of dripping colostrum or milk from the mammary glands, as noted in elephant birth protocols, or alveolar development in the mammary glands driven by progesterone (Senger, 2003). These changes may also initiate behaviors in herd mates that observe or sense changes in the pregnant dam, as secretions are known sources of chemo-signals (Rasmussen and Schulte 1998; Schulte \& Rasmussen 1999; Slade et al. 2003). Therefore, I hypothesized that changes in hormones could be detected in secretions from the temporal glands, mammary glands, vulva, and anus, and act as chemosignals to herd mates that parturition is imminent. To address this, I examined whether investigative behaviors 
directed towards the pregnant dam could be predictive of impending parturition in the Asian elephant.

Investigative behaviors included both self-directed behaviors and those sent from herd-mates towards the pregnant dam. Self-directed behaviors are most likely associated with physical changes in the pregnant dam, such as using the trunk to pull on swollen teats. Other-directed behaviors may stem from chemo-sensory signaling or other types of communication between herd mates, such as detecting changes in progesterone or cortisol.

\section{Methods}

\section{Subjects and Study Sites}

As noted in earlier chapters, we observed late gestation behavior in five Asian elephant pregnancies at the Oregon and Taronga Zoos. At the Oregon Zoo, we observed the same elephant during her nulliparous and primiparous pregnancies. At the Taronga Zoo, we observed three elephants during their nulliparous pregnancies. (See Table 1.2, Chapter 1).

\section{Data Collection}

Investigative behaviors selected for the ethogram were based on available birth protocols, conversations with elephant keeper and staff at the Oregon Zoo, and literature review (Rees 2009; Horback et al. 2014). The investigative category encompassed trunk-check behaviors, defined as an extension of the trunk towards specific areas of interest on the pregnant dam. These areas included the temporal gland, 
mammary glands, vulva and anus. To be coded as a trunk-check, the trunk had to extend to within 6 inches of the specified area. Trunk-check behaviors performed by the pregnant dam towards herself are "self-checks". Those performed by other elephants in the herd towards the pregnant dam are "herd mate-checks" (Table 4.1).

The design of the behavior study is given in Chapter 3. The "all-occurrences" sampling method was used for all trunk-check behaviors, as well as the preparturition behaviors (Martin \& Bateson 2007), and all behavioral data were collected at the same time.

\section{Analysis}

The data were partitioned into three time periods: Baseline, Pre and Post, based on the observed P4 drop in each elephant (See Chapter 2). As in Chapter 3, I analyzed two data sub-sets (binary data and non-zero data), followed by the full data set. All analyses were performed in SPSS version 22.0.

\section{Binary Data Set Analysis}

A likelihood ratio test was used to compare the presence or absence of each trunk-check behavior as a function of the time-partitioned periods (Baseline, Pre and Post).

Non-Zero Data Set Analysis

An ANOVA was used to compare the means for each trunk-check behavior among the Baseline, Pre and Post time periods. Data were examined for normality assumptions using the Shapiro-Wilk test. In cases where a significant result was found, a 
post-hoc Tukey test was used to determine which time periods differed significantly. A non-parametric Kruskal-Wallis test was also performed to compare the means for each trunk-check behavior among the Baseline, Pre and Post time periods. In cases where a significant result was found, mean ranks were compared using a multiple comparisons test.

\section{Full Data Set Analysis}

Prior to analysis, all trunk-check variables were examined for normality and variance assumptions. I used principal component analysis and step-wise reduction of generalized linear mixed models to evaluate these behaviors.

As in Chapter 3, a principal component analysis (PCA) was used to reduce the number of variables, or dimensions, to a fewer number of components. I retained components with an Eigen value greater than 1.0, or that fell above the elbow on the PCA scree plot for further modeling analyses. The PCA components were renamed by their highest loading variables.

Generalized Linear Mixed Models (GLMMs) with an identity link function and a normal probability distribution were used to further evaluate the data. A GLMM was used to determine what fixed effects and covariates, if any, accounted for the variance within a response, or dependent, variable. I first ran GLMM to examine each PCA component as a response variable. In each model, the time partition (Baseline, Pre and Post) was entered as a fixed effect categorical variable. Both P4 and cortisol percentage 
profiles were entered as continuous covariates. Elephant identity and institution were entered as random effects, to account for excess variability in the response variable.

Subsequently, GLMM were used to examine individual trunk-check behaviors as the response variable. Behaviors were selected that showed significance or trends in the binary or non-zero data set analyses. Fixed effects, covariates, and random effects remained as described above.

\section{Results}

\section{Binary Data Set}

I examined the presence or absence of each trunk-check behavior as a function of the time-partitioned data (Baseline, Pre and Post periods) using a likelihood ratio. No significant effects were found (Table 4.2).

\section{Non-Zero Data Set}

Variables in the non-zero data set did not meet normality assumptions as required for an ANOVA test as determined by the Shapiro-Wilk test for normality. Data transformations did not improve the Shapiro-Wilk statistics. However, an examination of the histograms and Q-Q plots of the transformed variables showed that many variables appeared normal, with slight positive skew. Because very small sample sizes can affect the outcome of the Shapiro-Wilk test, I ran an ANOVA (Table 4.3) and a nonparametric Kruskal-Wallis test (Table 4.4). 
An ANOVA compared the means for each trunk-check behavior among the three time periods (Table 4.3). No significant effects were found in any of the self- or herdmate trunk check behaviors towards the pregnant dam.

The non-parametric Kruskal-Wallis test showed no significant differences in the frequency of any self- or herd-mate trunk-check behaviors towards the pregnant dam among the Baseline, Pre and Post time periods.

\section{Full Data Set}

A PCA analysis identified two components (Table 4.5). Component 1 accounted for $39 \%$ of overall variance, and Component 2 accounted for an additional $22 \%$ of variance. In total, the two components accounted for $61 \%$ of the variance in the trunkcheck behavioral data. The variables with the highest loadings accounting for the greatest variance in Component 1 were Trunk-to-Vulva (Self), Trunk-to-Anus (Other), and Trunk-to-Mammary Glands (Other). This component was renamed the "Vulva-Self" variable, after the highest loading variable which was the main driver in addressing the greatest variance in the component. In Component 2, renamed the "Ear-Self" variable, the variables with the highest loadings were Trunk-to-Ear (Self), Trunk-to-Mammary Gland (Self), Trunk-to-Vulva (Other), and Trunk-to-Ear (Other).

I used GLMM to incorporate time partitions and endocrine profiles into our analyses. A separate GLMM was performed using each PCA component as a target to determine if time partitions and endocrine profiles accounted for the variance within each component. The model for the Vulva-Self Component was significant (Table 4.6). 
Additional GLMM models were run with each fixed effect tested separately, to determine which fixed effects had the greatest impact on the corrected model. After a step-wise reduction procedure, the best-fit model included both P4 and Cortisol as significant fixed effects (Table 4.7).

Additional GLMM models were performed for the Ear-Self component, and no significant fixed effects were found. In all models, random effects (Elephant ID and Institution) were significant, meaning there were significant differences in the PCA component values among elephants and between institutions. Thus, the variation among individuals and between institutions is high. The models accounted for this variation by holding these factors constant.

In addition to the PCA Component models, I ran GLMM models using individual behaviors as target variables to determine if time partitions and endocrine profiles account for the variance within each trunk-check variable (Tables 4.8-4.14). After a stepwise reduction procedure, I found the best-fit model for four significant fixed effects, as follows:

- Mammary Gland Self-Checks as a function of P4 levels (Table 4.10)

- Mammary Gland Other-Check as a function of P4 levels (Table 4.11)

- Vulva Self-Checks as a function of P4 and cortisol levels (Table 4.12)

- Anus Other-Check as a function of cortisol levels (Table 4.14) 


\section{Discussion}

It has been well documented that both male and female Asian elephants can successfully detect the estrous state of female elephants using the trunk to detect chemo-signals (Rasmussen et al. 1982; Schulte \& Rasmussen 1999; Slade et al. 2003; Sukumar 2003). However, to our knowledge, ours is the first study to evaluate the use of trunk-check behaviors to detect parturition status via chemo-signaling.

I evaluated the role of seven trunk-check behaviors in predicting parturition. I examined trunk-checks that were both self-directed and herd-mate directed towards the pregnant dam's ears, mammary glands, vulva and anus. (Note that the anus trunkcheck is herd-mate only, as an elephant cannot typically reach the anal region with their own trunk for a self-check). I report significant GLMMs incorporating Vulva-Self, AnusOther and Mammary-Other as a combined PCA target variable, with both P4 and cortisol as significant effects in the model. I found additional significant GLMMs using these same individual behaviors as target variables, as well as Mammary-Self. In each model, P4, cortisol, or both endocrine profiles had significant effects. In models where P4 was a predictor variable, the trunk-check behavior increased as P4 levels decreased. In models where cortisol was a predictor variable, the trunk-check behavior increased as cortisol levels increased. In both cases, Vulva Self-Checks, Anus Other-Checks, and Mammary Other-Checks varied significantly with endocrine profiles (P4, cortisol, or both). An additional individual behavior model was found for Mammary Gland Self-checks varying with P4. 
These results are a very promising indication that multiple trunk-check behaviors may be varying with the parturition status of the female as signaled by $\mathrm{P} 4$ and/or cortisol. However, I recognize that these models may not be practical predictive tools from a management standpoint, as with the Walking Backward behavior, I cannot predict the number of days to parturition based on a specific change in trunk-check behavior. However, keepers, veterinary staff, and other observes that are familiar with the regular behavioral repertoire of a pregnant female should pay close attention to these highlighted trunk-check behaviors. Keeping track of these behaviors, especially in conjunction with P4 and cortisol tracking, may help staff refine a window of expected parturition.

No significant models were found with the remaining three variables. No significant models were found that used either Self- or Other- Ear Trunk-Checks. However, according to Schulte \& Rasmussen (1999), female Asian elephants rarely secrete from their temporal glands, so there may not be any chemo-signaling opportunity. In addition, no significant models were found for Vulva-Other TrunkChecks. 
Table 4.1. Ethogram Variables for Trunk-Check Behaviors

\begin{tabular}{ll}
\hline Behavior & Description \\
\hline $\begin{array}{c}\text { Temporal Gland } \\
\text { Self } \\
\text { Other }\end{array}$ & $\begin{array}{l}\text { The pregnant dam extends trunk toward temporal gland by the ear on side of head } \\
\text { A herd mate extends trunk toward pregnant dam's temporal gland by the ear on } \\
\text { side of head }\end{array}$ \\
$\begin{array}{c}\text { Mammary Gland } \\
\text { Self }\end{array}$ & $\begin{array}{l}\text { The pregnant dam extend trunk towards mammary gland(s) located just behind } \\
\text { front legs } \\
\text { Other }\end{array}$ \\
$\begin{array}{c}\text { A herd mate extends trunk toward pregnant dam's mammary gland(s) on side of } \\
\text { head }\end{array}$ \\
$\begin{array}{l}\text { Self } \\
\text { Other }\end{array}$ & $\begin{array}{l}\text { The pregnant dam extend trunk towards vulva located between hind legs } \\
\text { A herd mate extends trunk toward pregnant dam's vulva located between hind legs }\end{array}$ \\
Onus & A herd mate extends trunk toward pregnant dam's anus located under the tail \\
\hline
\end{tabular}

Table 4.2. Likelihood Ratio Test for Trunk-Check Behaviors as a function of Partitioned Time (Baseline, Pre \& Post periods)

\begin{tabular}{ll}
\hline Variable & $\begin{array}{l}\text { Likelihood Ratio P- } \\
\text { values }\end{array}$ \\
\hline Trunk-Ear SELF & 0.153 \\
Trunk-Ear OTHER & 0.693 \\
Trunk-Mammary SELF & 0.266 \\
Trunk-Mammary OTHER & 0.056 \\
Trunk-Vulva SELF & 0.339 \\
Trunk-Vulva OTHER & 0.637 \\
Trunk-Anus OTHER & 0.583 \\
\hline
\end{tabular}


Table 4.3. ANOVA: Trunk-Check Behaviors in Baseline, Pre and Post time periods, with All Individuals Combined.

\begin{tabular}{llll}
\hline Variable & df & F-Statistic & Significance \\
\hline Trunk-Ear SELF & 2 & 0.147 & 0.864 \\
Trunk-Ear OTHER & 2 & 0.513 & 0.602 \\
Trunk-Mammary SELF & 2 & 2.509 & 0.091 \\
Trunk-Mammary OTHER & 2 & 0.308 & 0.738 \\
Trunk-Vulva SELF & 2 & 0.760 & 0.568 \\
Trunk-Vulva OTHER & 2 & 1.329 & 0.271 \\
Trunk-Anus OTHER & 2 & 1.619 & 0.217 \\
\hline
\end{tabular}

Table 4.4. Kruskal-Wallis Trunk Check Behaviors as a Function of Data Partition

\begin{tabular}{ll}
\hline Variable & $\begin{array}{l}\text { Kruskal-Wallis H- } \\
\text { Test Significance }\end{array}$ \\
\hline Trunk-Ear SELF & 0.799 \\
Trunk-Ear OTHER & 0.466 \\
Trunk-Mammary SELF & 0.208 \\
Trunk-Mammary OTHER & 0.809 \\
Trunk-Vulva SELF & 0.174 \\
Trunk-Vulva OTHER & 0.242 \\
Trunk-Anus OTHER & 0.388 \\
\hline
\end{tabular}

Table 4.5. Trunk-Check Variable Loadings by Component (highest loadings in bold).

\begin{tabular}{lcc}
\hline Variable & Component 1 & Component 2 \\
\hline Trunk-to-Ear (Self) & -.087 &. $\mathbf{8 7 9}$ \\
Trunk-to-Ear (Other) & .347 & .501 \\
Trunk-to-Mammary Glands (Self) & .027 & .761 \\
Trunk-to-Mammary Glands (Other) & .569 & .062 \\
Trunk-to-Vulva (Self) & .904 & .055 \\
Trunk-to-Vulva (Other) & .455 & .618 \\
Trunk-to-Anus (Other) & .898 & .125 \\
\hline
\end{tabular}

Table 4.6. GLMM PCA Component 1 with Data Partition, P4, and Cortisol as Fixed Effects ( $\left.{ }^{*} p<=0.05\right)$

\begin{tabular}{lllll}
\hline Source & F-Statistic & df1 & df2 & Significance \\
\hline Corrected Model & 2.421 & 4 & 150 & $0.051^{*}$ \\
Time Partition & 0.452 & 2 & 150 & 0.637 \\
P4 & 0.540 & 1 & 150 & 0.464 \\
Cortisol & 3.251 & 1 & 150 & 0.073 \\
\hline
\end{tabular}


Table 4.7. GLMM Models with PCA Component Vulva-Self and Each Fixed Effect $(* p<=0.05 ; * * p<=0.01)$

\begin{tabular}{lllll}
\hline Model & F-statistic & df1 & df2 & Significance \\
\hline Full Model: Vulva-Self $\sim$ Time Partition + P4 + Cortisol & 2.421 & 4 & 150 & $0.051^{*}$ \\
Sub Model: Vulva-Self Time Partition & 2.798 & 2 & 152 & 0.064 \\
Sub Model: Vulva-Self $\sim$ P4 + Cortisol & $\mathbf{4 . 4 6 6}$ & $\mathbf{2}$ & $\mathbf{1 5 2}$ & $\mathbf{0 . 0 1 3 ^ { * }}$ \\
Sub Model: Vulva-Self $\sim$ P4 & 5.982 & 1 & 153 & $0.016^{*}$ \\
Sub Model: Vulva-Self $\sim$ Cortisol & 6.836 & 1 & 153 & $0.010^{* *}$ \\
\hline
\end{tabular}

Table 4.8. GLMM Ear Self-Checks

\begin{tabular}{lllll}
\hline Model & F-statistic & df1 & df2 & Significance \\
\hline Full Model: Ear Self $\sim$ Time Partition + P4 + Cortisol & 0.810 & 4 & 150 & 0.521 \\
Sub Model: Ear Self Time Partition & 1.093 & 2 & 152 & 0.338 \\
Sub Model: Ear Self $\sim$ P4 + Cortisol & 0.406 & 2 & 152 & 0.667 \\
Sub Model: Ear Self $\sim$ P4 & 0.515 & 1 & 153 & 0.474 \\
Sub Model: Ear Self $\sim$ Cortisol & 0.063 & 1 & 153 & 0.802 \\
\hline
\end{tabular}

Table 4.9. GLMM Ear Other-Checks

\begin{tabular}{lllll}
\hline Model & F-statistic & df1 & df2 & Significance \\
\hline Full Model: Ear Other $\sim$ Time Partition + P4 + Cortisol & 0.205 & 4 & 150 & 0.935 \\
Sub Model: Ear Other $\sim$ Time Partition & 0.144 & 2 & 152 & 0.866 \\
Sub Model: Ear Other $\sim$ P4 + Cortisol & 0.211 & 2 & 152 & 0.810 \\
Sub Model: Ear Other $\sim$ P4 & 0.032 & 1 & 153 & 0.857 \\
Sub Model: Ear Other $\sim$ Cortisol & 0.265 & 1 & 153 & 0.607 \\
\hline
\end{tabular}

Table 4.10. GLMM Mammary Gland Self-Checks (*p<0.05) (Best Fit Model in Bold)

\begin{tabular}{lllll}
\hline Model & F-statistic & df1 & df2 & Significance \\
\hline $\begin{array}{l}\text { Full Model: Mammary Self } \sim \text { Time Partition + P4 + } \\
\text { Cortisol }\end{array}$ & 1.858 & 4 & 150 & 0.121 \\
Sub Model: Mammary Self Time Partition & & & & \\
Sub Model: Mammary Self $\sim$ P4 + Cortisol & 2.915 & 2 & 152 & 0.057 \\
Sub Model: Mammary Self P4 & 3.531 & 2 & 152 & $0.032^{*}$ \\
Sub Model: Mammary Self $\sim$ Cortisol & $\mathbf{6 . 4 4 5}$ & $\mathbf{1}$ & $\mathbf{1 5 3}$ & $\mathbf{0 . 0 1 2 *}$ \\
\hline
\end{tabular}

Table 4.11. GLMM Mammary Gland Other-Checks ( $\left.{ }^{*} p<0.05, * * p<0.01\right)$ (Best Fit Model in Bold)

\begin{tabular}{lllll}
\hline Model & F-statistic & df1 & df2 & Significance \\
\hline $\begin{array}{l}\text { Full Model: Mammary Other } \sim \text { Time Partition + P4 + } \\
\text { Cortisol }\end{array}$ & 2.694 & 4 & 150 & $0.033^{*}$ \\
Sub Model: Mammary Other $\sim$ Time Partition & 4.036 & 1 & 153 & $0.020^{*}$ \\
Sub Model: Mammary Other $\sim$ P4 + Cortisol & 5.005 & 2 & 152 & $0.008^{* *}$ \\
Sub Model: Mammary Other $\sim$ P4 & $\mathbf{1 0 . 2 6 9}$ & $\mathbf{1}$ & $\mathbf{1 5 3}$ & $\mathbf{0 . 0 0 2 * *}$ \\
Sub Model: Mammary Other $\sim$ Cortisol & 0.724 & 1 & 153 & 0.396 \\
\hline
\end{tabular}


Table 4.12. GLMM Vulva Self-Checks $\left({ }^{*} p<0.05,{ }^{* *} p<0.01\right)$ (Best Fit Model in Bold)

\begin{tabular}{lllll}
\hline Model & F-statistic & df1 & df2 & Significance \\
\hline Full Model: Vulva Self $\sim$ Time Partition + P4 + & 2.870 & 4 & 150 & $0.025^{*}$ \\
Cortisol & & & & \\
Sub Model: Vulva Self $\sim$ Time Partition & 3.621 & 2 & 152 & $0.029^{*}$ \\
Sub Model: Vulva Self $\sim$ P4 + Cortisol & $\mathbf{5 . 3 5 5}$ & $\mathbf{2}$ & $\mathbf{1 5 2}$ & $\mathbf{0 . 0 0 6 * *}$ \\
Sub Model: Vulva Self $\sim$ P4 & 6.526 & 1 & 153 & $0.012^{*}$ \\
Sub Model: Vulva Self $\sim$ Cortisol & 7.920 & 1 & 153 & $0.006^{* *}$ \\
\hline
\end{tabular}

Table 4.13. GLMM Vulva Other-Checks

\begin{tabular}{lllll}
\hline Model & F-statistic & df1 & df2 & Significance \\
\hline Full Model: Vulva Other Time Partition + P4 + & 0.511 & 4 & 150 & 0.728 \\
Cortisol & & & & \\
Sub Model: Vulva Other Time Partition & 0.605 & 2 & 152 & 0.547 \\
Sub Model: Vulva Other $\sim$ P4 + Cortisol & 0.388 & 2 & 152 & 0.679 \\
Sub Model: Vulva Other $~$ P4 & 0.597 & 1 & 153 & 0.441 \\
Sub Model: Vulva Other Cortisol & 0.014 & 1 & 153 & 0.905 \\
\hline
\end{tabular}

Table 4.14. GLMM Anus Other-Checks $\left({ }^{*} p<0.05,{ }^{* *} p<0.01\right)$ (Best Fit Model in Bold)

\begin{tabular}{lllll}
\hline Model & F-statistic & df1 & df2 & Significance \\
\hline Full Model: Anus Other Time Partition + P4 + & 2.708 & 4 & 150 & $0.032^{*}$ \\
Cortisol & & & & \\
Sub Model: Anus Other Time Partition & 2.562 & 2 & 152 & 0.081 \\
Sub Model: Anus Other $\sim$ P4 + Cortisol & 5.075 & 2 & 152 & $0.007^{* *}$ \\
Sub Model: Anus Other $\sim$ P4 & 4.250 & 1 & 153 & $0.041^{*}$ \\
Sub Model: Anus Other Cortisol & $\mathbf{9 . 2 4 0}$ & $\mathbf{1}$ & $\mathbf{1 5 3}$ & $\mathbf{0 . 0 0 3 * *}$ \\
\hline
\end{tabular}




\section{Chapter 5: Activity Budget}

\section{Introduction}

Daily activity budgets may be impacted by changes in some behaviors, especially as noted in birth protocols (see Table 1.1) such as increased pacing, reduction of appetite/eating, and increased urination and defecation (see Table 1.1). An ideal comparison of activity would be between an elephant's budget during the preparturient period and a period when not pregnant. However, the data set for the current study was focused solely on the preparturient period. Therefore, my primary objective in this chapter is to summarize the activity budget of the focal elephants during the weeks prior to parturition. I make generalized comparisons of these late-gestation activity budgets to published activity budgets of non-pregnant Asian elephants. In addition, this unique data set also allowed for the comparison of two different sampling techniques used in activity budget data collection: one-zero and instantaneous sampling. The effect of the different sampling techniques on behavior allocation within an activity budget is discussed.

\section{Methods}

\section{Subjects and Study Sites}

Subjects and study sites were as previously described. (See Table 1.2, Chapter 1).

\section{Data Collection}

The activity budget behaviors selected for the ethogram were based on published activity budgets (Rees 2009; Horback et al. 2014), conversations with zoo 
keepers and staff, and initial observation of the pregnant Oregon Zoo elephant. The behaviors and their description are provided in Table 5.1.

Both "one-zero" and "instantaneous" sampling methods were used for activity budget behaviors (Martin \& Bateson 2007). The Oregon Zoo followed a one-zero protocol, with observers recording behaviors as they occurred during each 2-minute interval, for a total of 15 intervals in each 30-minute observation. In one-zero sampling, if the behavior occurs during the 2 minute time interval a " 1 " is recorded. If the behavior continues into the next time interval, a "1" is also placed in that 2-minute time interval. If the behavior does not occur during a 2-minute time interval, the interval is left blank and a " 0 " is later recorded. Taronga Zoo typically followed an instantaneous protocol, with observers recording the behavior observed at 2-minute intervals, on the instant. In instantaneous sampling, only one behavior is recorded for each interval. An exception to the typical sampling protocol used at Taronga Zoo was the data collection for PakBoon in which both one-zero and instantaneous sampling protocols were used. I reviewed each record for Pak Boon and categorized it as either one-zero or instantaneous. In order to analyze all activity budget data collected, I created two data sets. One data set was for the one-zero sampling method that included Rose Tu's data from the Oregon Zoo and part of Pak Boon's data from Taronga. The second data set was for the instantaneous sampling method that included Porntip and Thong Dee's data, and a partial data set for Pak Boon, all from the Taronga Zoo. 


\section{Analysis}

One-Zero Data Set

Some variables were aggregated for analysis (Table 5.2). Initially, the locomotion category encompassed three variables- Stationary, Walking and Pacing. For analysis, these were re-categorized so that Walking and Pacing were grouped in a locomotion category, while Stationary became the sole variable in its own category. The variable Lean was removed from the one-zero dataset, as the self-directed category was deemed inappropriate for this variable. Furthermore, it was presumed there was significant overlap between Lean and Stationary as they are expected to co-occur. To verify this presumption, the original data from RoseTu's 2012 pregnancy was reviewed prior to daily aggregation. Of 205 instances of Lean behavior, 202 records (98.5\%) were also marked as Stationary. Therefore, it was concluded that Stationary already captured the vast majority of instances of Lean.

The elimination (urination and defecation) category was also removed from the one-zero dataset because the occurrence was low, it was not a behavior that appeared in activity budgets, and I presumed significant overlap with Stationary. Also, when the data from RoseTu's 2012 pregnancy were reviewed, urination occurred in $2.2 \%$ of records and of these $93.8 \%$ were also recorded as Stationary. Defecation occurred in $2.0 \%$ of records, and of these, $96.5 \%$ were also recorded as Stationary. Due to the low occurrence, and the overlap with Stationary behavior, the elimination variables were removed from the dataset. 
All variables were examined for assumptions of normality and equal variance, and data transformations were performed where necessary. Data were summarized in proportion of daily time spent in each behavior, as well as average daily occurrence values. Further analyses used an ANOVA to compare means of each budget category behaviors across elephants. Where a significant difference was found, post hoc Tukey tests were performed. Instantaneous Data Set

Some variables were aggregated for analysis (Table 5.3). As with the one-zero data set, the initial locomotion category encompassed three variables- Stationary, Walking and Pacing. For analysis, these were re-categorized so that Walking and Pacing were grouped in a locomotion category, while Stationary became the sole variable in its own category. As discussed above, Self-directed was deemed an inappropriate categorization for Lean, and in the one-zero data set Lean and Stationary showed significant overlap. Thus, for the instantaneous data set, all records of Lean were added to the Stationary category. The Elimination category was also added to Stationary in the instantaneous dataset. It was not necessary as an independent category, since the occurrence was low and it was not a behavior that typically appears in activity budgets. Since I determined there was significant overlap between the elimination variables and Stationary in the one-zero dataset, I added Urination and Defecation to the stationary category in the instantaneous dataset. The locomotion category encompassed walking and pacing, as with the one-zero data set. 
All variables were examined for assumptions of normality and equal variance, and data transformations were performed where necessary. Data were summarized in proportion of daily time spent in each behavior, as well as average daily occurrence values. Further analyses used an ANOVA to compare means of each budget category behaviors across elephants. Where a significant difference was found, post hoc Tukey tests were performed.

\section{Results}

Due to scheduling issues and occasional limits on access to the elephants, all four observation periods were not always completed each day (Table 5.4). The average number of records analyzed for each elephant each day fell between 3.1 and 3.9.

\section{One-Zero Data Set}

I evaluated Stationary; Locomotion, (including Walking and Pacing); SelfDirected; Feeding, (including Eating/Manipulating Food and Drinking); and Other, (including Keeper Modified and Not Visible) behaviors. Activity budget results display the proportion of observations during which each behavior category was observed (Figure 5.1) and the average daily occurrences of each behavior (Table 5.5). One-zero sampling does not assume mutually exclusivity among categories, since all behaviors observed during an interval are recorded.

The activity budgets using one-zero sampling revealed similar patterns among the three elephants. Stationary behavior was observed most often with a mean proportion of 0.45 , followed by feeding behavior with a mean proportion of 0.28 . 
An ANOVA with post-hoc t-test was performed on the one-zero data set. The ANOVA showed all categories had a significant mean difference between at least two elephants ( $p<0.01$ in all categories) (Table 5.6). Further post-hoc testing found 10 pairwise comparisons with significantly different means (Table 5.7). Locomotion was significantly different among all three pairwise comparisons $(p<0.01)$. The stationary variable was significantly different between PakBoon and RoseTu in both $2008(p<0.01)$ and 2012 ( $p<0.01)$. Self-directing showed a significant difference between PakBoon and RoseTu in 2012 ( $p<0.05)$. Feeding showed a significant difference between RoseTu in 2008 and PakBoon $(p<0.05)$ and RoseTu in 2008 and $2012(p<0.01)$. Finally, the Other category showed a significant difference between PakBoon and RoseTu in both 2008 $(p<0.01)$ and $2012(p<0.01)$.

\section{Instantaneous Data Set}

I evaluated Stationary, (including Stationary, Lean and Elimination); Locomotion, (including Walking and Pacing); Self-Directed; Feeding, (including Eating/Manipulating Food and Drinking); and Other, (including Keeper Modified and Not Visible) behaviors. Activity budget results display the proportion of observations during which each behavior category was observed (Figure 5.2), and the average number of occurrences of each behavior (Table 5.8). For instantaneous sampling, the resulting proportions assume behaviors are mutually exclusive, which is not necessarily true given some behaviors can co-occur (such as feeding and stationary behavior). 
The activity budgets using instantaneous sampling revealed similar patterns among the three elephants. The largest proportion of time was spent feeding with a mean proportion of 0.42 , followed by stationary behavior with a mean proportion of 0.25. Since these two behaviors are not naturally mutually exclusive, feeding behavior was prioritized over stationary behavior in data recording. All elephants were more often observed in stationary behavior $(M=0.24)$ than locomotor behavior $(M=0.14)$.

An ANOVA and post-hoc t-test were also performed on the instantaneous data set. The ANOVA showed two categories, locomotion and feeding, had a significant mean difference between at least two elephants $(p=0.002$ and $p=0.007$, respectively; Table 5.9). Further post-hoc testing found that Locomotion and Feeding showed a significant difference between PakBoon(Inst) and Porntip ( $p<0.01$ and $p<0.05$, respectively; Table 5.10).

\section{Discussion}

All-occurrence sampling provides true frequencies (or very close approximations of true frequencies) of behaviors and is an ideal method for determining activity budgets (Martin \& Bateson 2007). However, all-occurrence sampling is time consuming, and incompatible with large ethograms in which a number of behavior categories and/or animals are observed. The instantaneous sampling method we utilized has been shown to estimate the proportion of time spent in an activity (Dunbar, 1976; Leger, 1977). While one-zero sampling tends to over-estimate the duration and under-estimate the number of bouts of behaviors, it can be useful for behaviors that are intermittent 
(Martin \& Bateson 2007). In both instantaneous and one-zero sampling, the length of the interval needs to account for the general length of time spent in specific behaviors, as well as to balance the complexity of the ethogram. While shorter time intervals are ideal to capture most behaviors and provide an accurate estimate of time budgets, short time intervals are not always practical with a complex ethogram or with large animals with slower-paced behaviors. For this study, the activity budget behaviors were only a portion of the overall observed behaviors, which included a number of preparturition behaviors and social interactions. Shorter time intervals would have made it difficult to observe all of the behaviors included in the ethogram. And because elephants are large and tend to require more time to complete behaviors, a longer time interval was more appropriate (Rees 2009). Therefore, an interval of 2 minutes was selected to balance the needs of the study with the desire to capture as many behaviors as possible.

In comparing results from both the instantaneous and one-zero sampling techniques, the activity budgets align with published data reporting results from instantaneous sampling. Rees (2009) reported feeding behavior $27.4-41.4 \%$ of the time, as compared with our average feeding proportions of $27 \%$ (one-zero) and $42 \%$ (instantaneous). Stationary behavior was recorded $22.9-42 \%$ of the time, as compared to our average stationary proportions of $24 \%$ (one-zero) and $43 \%$ (instantaneous). Rees observed walking behavior $6.1-19.2 \%$ of the time. Our locomotor observations were on the higher end of this range at 14\% (instantaneous), and 16\% (one-zero), but included pacing behavior. 
One study utilizing instantaneous sampling (Horback et al. 2014) reported foraging (mean=52\%) as the most common behavior recorded, followed by resting (mean=31.3\%) and then walking (mean=9.2\%). This hierarchy of behavior is consistent with our results from instantaneous sampling, with feeding being the most frequent behavior (42\%), followed by stationary (24\%) and locomotion (14\%). While the percentages vary due to the inclusion of different categories in the Horback study, the pattern for these three main behaviors is very similar.

Of note is the relatively high feeding rate of PakBoon, with a proportion of $50 \%$ and $34 \%$ for instantaneous and one-zero sampling, respectively. The data were examined for unusual data collection patterns, such as one observer consistently scoring higher feeding rates than others, as well as for temporal changes in feeding, such as significantly higher feeding rates as parturition approached. No aberrations were observed. I therefore conclude that Pak Boon had higher than typical feeding rates. While I was unable to make the ideal comparison of intra-elephant behavior while not pregnant to behavior in late gestation, these summary data do provide a starting point for future work. In addition, I was able to highlight differences observed in overall budget allocation depending on the sampling method used. For future work which focuses solely on activity budget behavior, I recommend the instantaneous sampling method. It is more often reported in published reports, which allows for better comparison. Instantaneous sampling is also a better estimate of the overall portion of time spent in various activities. 


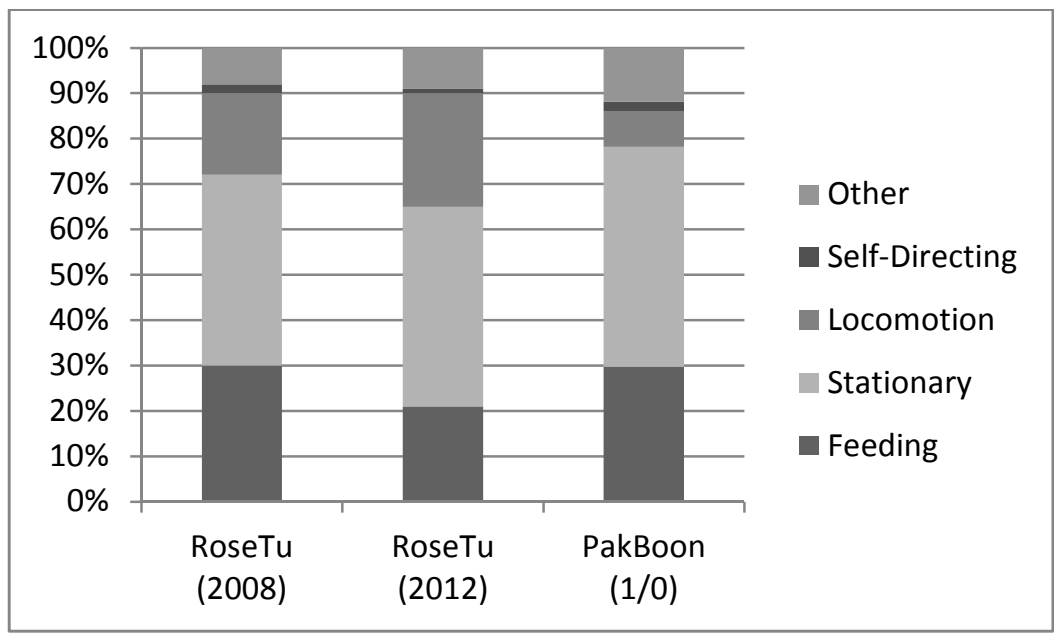

Figure 5.1. Average daily proportion of intervals during which each behavior was observed (one-zero sampling).

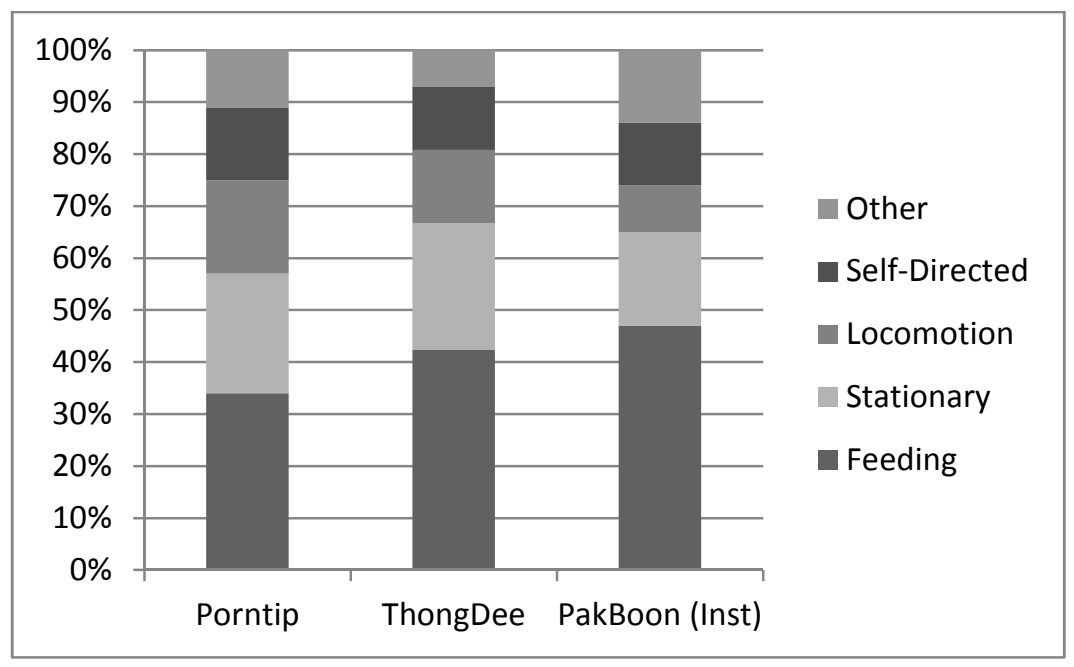

Figure 5.2. Average daily proportion of time observed in each behavior category (instantaneous sampling). 
Table 5.1. Ethogram of Activity Budget Behaviors

\begin{tabular}{|c|c|}
\hline Behavior & Description \\
\hline \multicolumn{2}{|l|}{ Locomotion } \\
\hline Stationary & $\begin{array}{l}\text { Not deliberately moving forward, backward or sideways for more than } 3 \\
\text { seconds without interruption. }\end{array}$ \\
\hline Walking & $\begin{array}{l}\text { Any movement that transports elephant forward, backward or sideways for } \\
3 \text { seconds or more. }\end{array}$ \\
\hline Pacing & $\begin{array}{l}\text { For } 3 \text { or more repetitions: Walking from one point to another, stopping, } \\
\text { turning and walking back to the starting point, OR walking in a loop. }\end{array}$ \\
\hline \multicolumn{2}{|l|}{ Self-Directed } \\
\hline Sucking Thumb & Holds side or end of own trunk in own mouth for 3 or more seconds. \\
\hline Lean & $\begin{array}{l}\text { Weight is placed on another individual or fixed object; rests while standing. } \\
\text { This includes body and/or head, but does not include resting on trunk (i.e. } \\
\text { using trunk as a "crutch"). }\end{array}$ \\
\hline \multicolumn{2}{|l|}{ Elimination } \\
\hline Urination & Any elimination of urine \\
\hline Defecation & Any elimination of solid waste \\
\hline \multicolumn{2}{|l|}{ Feeding } \\
\hline Eating/Manipulating & $\begin{array}{l}\text { Consumption of any food item, or using trunk or mouth to manipulate food } \\
\text { or browse. }\end{array}$ \\
\hline Drinking & Consumption of water. Trunk goes from a water sources directly to mouth. \\
\hline \multicolumn{2}{|l|}{ Other } \\
\hline Keeper Modified & $\begin{array}{l}\text { Keeper is present and elephant is waiting for, or responding to, keeper } \\
\text { instruction. Continue observing and recording other behaviors. }\end{array}$ \\
\hline Not Visible & $\begin{array}{l}\text { Elephant is not visible (out of sight), or is partially visible and observation of } \\
\text { behaviors may be obstructed, for more than } 30 \text { seconds within the two } \\
\text { minute time interval. }\end{array}$ \\
\hline
\end{tabular}


Table 5.2. Variable aggregation for analysis of data collected using one-zero sampling

\begin{tabular}{|c|c|}
\hline Behavior & Description \\
\hline \multicolumn{2}{|l|}{ Stationary } \\
\hline Stationary & $\begin{array}{l}\text { Not deliberately moving forward, backward or sideways for more than } 3 \\
\text { seconds without interruption. }\end{array}$ \\
\hline \multicolumn{2}{|r|}{ 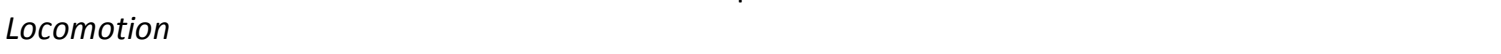 } \\
\hline Walking & $\begin{array}{l}\text { Any movement that transports elephant forward, backward or sideways for } 3 \\
\text { seconds or more. }\end{array}$ \\
\hline Pacing & $\begin{array}{l}\text { For } 3 \text { or more repetitions: Walking from one point to another, stopping, } \\
\text { turning and walking back to the starting point, OR walking in a loop. }\end{array}$ \\
\hline \multicolumn{2}{|r|}{ 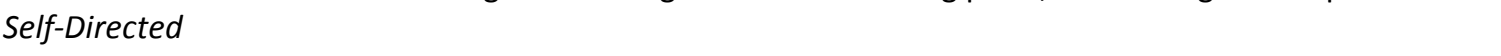 } \\
\hline Sucking Thumb & Holds side or end of own trunk in own mouth for 3 or more seconds. \\
\hline \multicolumn{2}{|l|}{ Feeding } \\
\hline Eating/Manipulating & $\begin{array}{l}\text { Consumption of any food item, or using trunk or mouth to manipulate food or } \\
\text { browse. }\end{array}$ \\
\hline Other & Consumption of water. Trunk goes from a water sources directly to mouth. \\
\hline Keeper Modified & $\begin{array}{l}\text { Keeper is present and elephant is waiting for, or responding to, keeper } \\
\text { instruction. Continue observing and recording other behaviors. }\end{array}$ \\
\hline Not Visible & $\begin{array}{l}\text { Elephant is not visible (out of sight), or is partially visible and observation of } \\
\text { behaviors may be obstructed, for more than } 30 \text { seconds within the two } \\
\text { minute time interval. }\end{array}$ \\
\hline
\end{tabular}


Table 5.3. Variable aggregation for analysis of data collected using instantaneous sampling

\begin{tabular}{|c|c|}
\hline Behavior & Description \\
\hline \multicolumn{2}{|l|}{ Stationary } \\
\hline Stationary & $\begin{array}{l}\text { Not deliberately moving forward, backward or sideways for more than } 3 \\
\text { seconds without interruption. }\end{array}$ \\
\hline Lean & $\begin{array}{l}\text { Weight is placed on another individual or fixed object; rests while standing. } \\
\text { This includes body and/or head, but does not include resting on trunk (i.e. } \\
\text { using trunk as a "crutch"). }\end{array}$ \\
\hline Elimination & Any elimination of urine or solid waste \\
\hline \multicolumn{2}{|l|}{ Locomotion } \\
\hline Walking & $\begin{array}{l}\text { Any movement that transports elephant forward, backward or sideways for } \\
3 \text { seconds or more. }\end{array}$ \\
\hline Pacing & $\begin{array}{l}\text { For } 3 \text { or more repetitions: Walking from one point to another, stopping, } \\
\text { turning and walking back to the starting point, OR walking in a loop. }\end{array}$ \\
\hline \multicolumn{2}{|l|}{ Self-Directed } \\
\hline Sucking Thumb & Holds side or end of own trunk in own mouth for 3 or more seconds. \\
\hline \multicolumn{2}{|l|}{ Feeding } \\
\hline Eating/Manipulating & $\begin{array}{l}\text { Consumption of any food item, or using trunk or mouth to manipulate food } \\
\text { or browse. }\end{array}$ \\
\hline Drinking & Consumption of water. Trunk goes from a water sources directly to mouth. \\
\hline \multicolumn{2}{|l|}{ Other } \\
\hline Keeper Modified & $\begin{array}{l}\text { Keeper is present and elephant is waiting for, or responding to, keeper } \\
\text { instruction. Continue observing and recording other behaviors. }\end{array}$ \\
\hline Not Visible & $\begin{array}{l}\text { Elephant is not visible (out of sight), or is partially visible and observation of } \\
\text { behaviors may be obstructed, for more than } 30 \text { seconds within the two } \\
\text { minute time interval. }\end{array}$ \\
\hline
\end{tabular}


Table 5.4. Count of Records Used in Activity Budget Analysis

\begin{tabular}{lllll}
\hline Institution & Elephant & $\begin{array}{l}\text { Number of Records } \\
\text { Utilized for Activity } \\
\text { Budget Analysis }\end{array}$ & Number of Days & $\begin{array}{l}\text { Average Number } \\
\text { of Records per } \\
\text { Day }\end{array}$ \\
\hline Oregon Zoo & RoseTu (2008) & 122 & 34 & 3.6 \\
& RoseTu (2012) & 192 & 51 & 3.8 \\
Taronga Zoo & PakBoon (1/0) & 46 & 15 & 3.1 \\
& PakBoon (Inst) & 72 & 23 & 3.1 \\
& PornTip & 128 & 37 & 3.5 \\
& Thong Dee & 120 & 31 & 3.9 \\
\hline
\end{tabular}

Table 5.5. Average daily occurrence of each behavioral category (One-Zero Sampling).

\begin{tabular}{lccccc}
\hline & Stationary & Locomotion & Self-Directed & Feeding & Other \\
\hline RoseTu (2008) & $14.91(0.40)$ & $6.30(1.51)$ & $5.11(3.02)$ & $10.42(5.18)$ & $2.89(2.65)$ \\
RoseTu (2012) & $13.91(1.28)$ & $7.85(2.02)$ & $0.31(0.53)$ & $6.74(2.89)$ & $2.82(1.83)$ \\
PakBoon (1/0) & $7.47(4.74)$ & $1.74(0.73)$ & $1.28(1.72)$ & $7.27(1.81)$ & $2.51(1.38)$ \\
\hline Average (SD): & $13.99(1.37)$ & $6.44(2.67)$ & $0.49(0.59)$ & $8.10(4.11)$ & $2.89(2.19)$ \\
\hline
\end{tabular}

Table 5.6. All behavioral categories showed significant mean differences between at least two elephants $(* * p<0.01)($ One-Zero)

\begin{tabular}{llll}
\hline & F-Statistic & df & Significance \\
\hline Stationary & 48.076 & 2 & $0.000^{* *}$ \\
Locomotion & 74.195 & 2 & $0.000^{* *}$ \\
Self-Directing & 4.873 & 2 & $0.010^{* *}$ \\
Feeding & 8.968 & 2 & $0.000^{* *}$ \\
Other & 7.391 & 2 & $0.001^{* *}$ \\
\hline
\end{tabular}

Table 5.7. Tukey's HSD mean difference scores and significance levels $\left({ }^{*} p<0.05 ;{ }^{* *} p<0.01\right)(O n e-Z e r o)$

\begin{tabular}{llllll}
\hline & Stationary & Locomotion & Self-Directing & Feeding & Other \\
\hline RoseTu08 vs RoseTu12 & 0.93 & $-1.58^{* *}$ & 0.43 & $3.65^{* *}$ & 0.05 \\
RoseTu12 vs PakBoon (1/0) & $4.39^{* *}$ & $6.06^{* *}$ & $-0.90^{*}$ & -0.71 & $2.14^{* *}$ \\
PakBoon (1/0) vs RoseTu08 & $-5.32^{* *}$ & $-4.49^{* *}$ & 0.46 & $-2.94^{*}$ & $-2.19^{* *}$ \\
\hline
\end{tabular}


Table 5.8. Average number of occurrences observed using instantaneous sampling

\begin{tabular}{lccccc}
\hline & Stationary & Locomotion & Self-Directed & Feeding & Other \\
\hline Porntip & 3.50 & 2.68 & 2.03 & 5.14 & 1.60 \\
ThongDee & 3.65 & 2.07 & 1.85 & 6.33 & 1.10 \\
PakBoon (Inst) & $3.41(2.08)$ & $1.35(0.80)$ & $1.84(2.23)$ & $6.28(1.52)$ & $1.97(1.47)$ \\
\hline Average (SD): & $3.35(1.95)$ & $2.14(1.46)$ & $1.89(1.81)$ & $5.97(2.33)$ & $1.52(1.31)$ \\
\hline
\end{tabular}

Table 5.9. Locomotion and Feeding behavioral categories showed significant mean differences between at least two elephants $\left({ }^{*} p<0.05,{ }^{*} p<0.01\right)$ (Instantaneous)

\begin{tabular}{llll}
\hline & F-Statistic & df & Significance \\
\hline Stationary(Log) & $4.117^{*}$ & 2 & 0.020 \\
Locomotion & $6.683^{* *}$ & 2 & 0.002 \\
Self-Directing(Log) & 0.992 & 2 & 0.375 \\
Feeding & $5.191^{* *}$ & 2 & 0.007 \\
Other(SqRt) & $3.939 *$ & 2 & 0.023 \\
\hline
\end{tabular}

Table 5.10. Tukey's HSD mean difference scores and significant levels $\left({ }^{*} p<0.05 ; * * p<0.01\right)$ (Instantaneous)

\begin{tabular}{lllll}
\hline & Stationary(Log) & Locomotion & Feeding & Other(SqRt) \\
\hline ThongDee vs Porntip & 0.012 & -.557 & $1.376^{*}$ & -0.292 \\
ThongDee vs PakBoon (Inst) & $0.149 *$ & 0.764 & -0.235 & $-0.379^{*}$ \\
Porntip vs. PakBoon (Inst) & $0.136^{*}$ & $1.322^{* *}$ & $-1.610^{*}$ & -0.087 \\
\hline
\end{tabular}




\section{Chapter 6: Conclusions}

The ability to accurately predict parturition in the captive Asian elephant is imperative for improving birth outcomes and moving towards a self-sustaining population (Szdzuy et al. 2006). With an aging female population, low fecundity rates and high calf mortality, attaining self-sustaining populations remains a challenge (Taylor \& Poole 1998; Szdzuy et al. 2006; Wiese 2000; Rees 2003; Brown et al. 2004b). Complications, including stillbirth, protracted labor, dystocia and maternal aggression towards a newborn calf (Taylor \& Poole 1998; Szdzuy et al. 2006, Thitaram et al. 2006; Schaftenaar 2013) can require immediate intervention by keepers and veterinary staff. Predicting when a calf is expected to arrive can aid in recognizing and ameliorating these complications.

The aim of this study was to evaluate the efficacy of behaviors and endocrine patterns in the preparturient period as tools for predicting parturition. Finding tools that can aid in predicting parturition in captive Asian elephants can benefit all institutions that house them, either as a supplement to current endocrine methods, or as a separate tool when endocrine profiling is not available. Regular, frequent monitoring of progesterone has been a useful tool in determining the timing of parturition (Brown \& Lehnhardt 1995; Brown 2000; Brown et al. 2004a; Schaftenaar \& Hildebrandt 2006), but I report this tool is not without limitations. I assessed the role of behaviors as predictors of parturition both independent of and in conjunction with endocrine profiles. 


\section{The Role of Progesterone and Cortisol as Predictors of Parturition}

I report expected late-gestation endocrine patterns in all five pregnancies. There was a drop in progesterone, with P4 levels being lowest in the last few days before parturition. I also observed significantly higher levels of cortisol during this same time frame.

The mechanisms involved in maternal late-gestation hormone profiles in the Asian elephant are not well understood and deserve further study. The role of the fetus in triggering a cascade of events that initiates parturition in the mother have been studied in sheep (Rees et al. 1975, Challis et al. 2000), pigs (Kattesh et al. 1997), and the wallaby (Ingram et al. 1999). The activation of the fetal hypothalamic-pituitary-adrenal (HPA) axis and a subsequent increase in fetal cortisol caused an observable surge in assessed levels of maternal circulating cortisol in the ewe (Challis et al. 2000). Meyer et al. (2004) hypothesize that a similar surge in elephant maternal cortisol that has been observed 8 to 11 days prior to parturition may also have a fetal origin and play a role in initiating parturition. Fetal cortisol may then induce conversion of maternal progesterone to estradiol via normal endocrine actions (Senger 2003). If the role of fetal HPA activation and fetal cortisol surges are validated in the Asian elephant, these changes may account for the drastic drop observed in circulating maternal progesterone that precedes parturition.

I also report limitations in the use of P4 and cortisol in predicting parturition. In our sample of just five pregnancies, three of the elephants showed P4 profiles that 
differed from the norm in some way: one elephant showing a P4 drop 10 days prior to parturition, well outside the expected 2-5 day window (Brown 2000; Meyer et al. 2004); two elephants showed increasing P4 after the observed drop. I also observed high intraand inter-individual variation in cortisol, with one elephant showing considerable surges up to three weeks prior to parturition. Thus, it may be difficult to isolate parturitionrelated surges in cortisol from other stress-related surges. Furthermore, there are limitations in comparing results based on assays with different antibodies and different cross reactivities (see Chapter 2). While these limitations did not affect the overall endocrine patterns observed across all five pregnancies, they can make it difficult to detect the true drop in $\mathrm{P} 4$ and the true surges in cortisol in real time, and therefore to accurately predict parturition.

Late gestation markers should not be limited to P4 and cortisol. Meyer et al. (2004) examined levels of prolactin and relaxin by month of gestation. Further work could examine late-gestation profiles of prolactin and relaxin with more frequent monitoring. The protein ceruloplasmin has been used as a pregnancy marker in pandas (Willis et al. 2011). To my knowledge, ceruloplasmin has not yet been examined in the Asian elephant and should be considered as a possible marker during pregnancy and/or late gestation.

In addition to limitations in tracking P4, cortisol, and other endocrine profiles, there are institutions that do not regularly perform endocrine monitoring of their herds 
(Brown et al. 2004b). This highlights the need for additional markers, i.e. behaviors, to support late gestation management.

\section{The Role of Preparturition Behavior as Predictors of Parturition}

I evaluated the role of twelve preparturition behaviors in predicting parturition. I report an increase in Walking Backward behavior across the three time periods (Baseline, Pre and Post) as parturition approached. This result was also supported in a GLMM model in which walking backwards varied across these three time periods. An additional model found a significant relationship between levels of P4 and the Walking Backward behavior. I recognize limitations in using this as a predictive model, since it cannot be used to predict an accurate date of parturition. However, I recommend that keepers, veterinary staff, and other observers who are familiar with the regular behavioral repertoire of a pregnant female pay close attention to the walking backward behavior. Keeping track of this behavior, especially in conjunction with P4 tracking, can help staff provide a better window of expected parturition.

The specific purpose for the Walking Backwards behavior is unknown, despite previous observations of this behavior in the preparurient period (Leuthold \& Leuthold 1975). In another quadruped mammal, the cat, walking backwards creates flexion of the spine, versus a straight spine in forward walking movement, and shifts the major swinging movement in the hind limbs from the knee in forward walking to the hip in backward walking (Buford et al. 1990). These observations of posture and hind limb movement in another quadruped may lend support for our observations of this 
behavior in late gestation in the Asian elephant. The research on backwards walking in quadruped cats suggests that shifting to walking backwards in itself changes the curve of the spine, and it is feasible that this could be a technique to allow the calf to optimize its position. In addition, the observations on hind limb movement in quadrupeds may support our earlier proposal that walking backwards could play a role in reducing or changing stress on the joints. Having more "swing" in the leg come from the hip versus the knee could temporarily reduce stress in hind-limb knee joints. Although posture and limb movement of the digitigrade cat may differ from the large-bodied, semi-digitigrade elephant, it is important to note that Ren et al. (2008) concluded that elephant limb motion is more similar to smaller animals than previously thought. Thus, previous work on limb movement and backwards gate in other mammals should not be discounted. Future work should further evaluate the mechanics of Asian elephant limb movement, as well as elucidate possible advantages to walking backwards in late gestation. Given our observations in this study, I propose that walking backwards may be an attempt for the pregnant dam to find higher ground for the hind limbs in order to relieve pressure on the pelvic rim and allow optimal positioning of the calf.

Of note are the eleven preparturition behaviors that did not show any significant change over the three time periods, or with changes in $\mathrm{P} 4$ or cortisol profiles. There are a number of possible conclusions we can draw from this. The first is that these behaviors simply are not associated with late gestation or parturition. While this is possible, I suggest a more nuanced approach to these behaviors. It is possible that we 
simply did not observe behavioral changes that are actually present. For example, Szdzuy et al. (2006) evaluated behaviors at night because all study subjects had similar handling practices during night time hours, and they experienced fewer disturbances at night. Night observations were selected for proximate reasons to address research design. However, I propose that the researchers may have touched upon an ultimate, evolutionary role for night time behaviors: behaviors performed at night are not easily observed by others, especially predators. Due to their sheer size, healthy adult Asian elephants have no natural predators (Fernando \& Lande 2000; Sukumar 2003). Newborn calves, however, are vulnerable to attack from large predators (Thuppil \& Coss 2013). Thus, masking signs of parturition may be an evolutionary tool for predator avoidance. Elephants may have multiple methods for "hiding" parturition. One would be to limit obvious signs of labor. This could explain why our preparturition behavior results are limited to just one behavior (walking backwards). Since an important strategy may be to perform preparturition-related behaviors in the cover of night, as Szdzuy et al. (2006) observed in the previously discussed study, I recommend further evaluation of nighttime behaviors.

\section{The Role of Trunk-Check Behavior as Predictors of Parturition}

I evaluated the role of seven trunk-check behaviors in predicting parturition. I examined trunk-checks that were both self-directed and herd-mate directed towards the pregnant dam's ears (near the temporal glands), mammary glands, vulva and anus. I found a significant GLMM model that used the first PCA component, Vulva-Self, as a 
target to determine whether time partitions and endocrine profiles account for the variance within each component. A step-wise reduction method resulted in retaining both P4 and cortisol as significant effects in this model.

I found additional significant GLMM models using individual behaviors as target variables to determine if time partitions and endocrine profiles account for the variance within each trunk-check variable.

- Mammary Gland Self-Checks as a function of P4 levels

- Mammary Gland Other-Check as a function of P4 levels

- Vulva Self-Checks as a function of P4 and cortisol levels

- Anus Other-Check as a function of cortisol levels

These individual behavior GLMM models aligned well with the significant results from the Vulva-Self (PCA Component 1) GLMM, above. In both cases, Vulva Self-Checks, Anus Other-Checks, and Mammary Other-Checks varied significantly with endocrine profiles (P4, cortisol, or both). An additional individual behavior model was found for Mammary Gland Self-checks varying with P4. It has been well documented that both male and female Asian elephants detect chemo-signals in other elephants using investigative trunk behaviors. Male Asian elephants can successfully detect the reproductive status of females (Sukumar 2003); and female Asian elephants can successfully detect the reproductive status of male (Schulte \& Rasmussen 1999, Sukumar 2003) and female (Slade et al. 2003; Sukumar 2003). Furthermore, previous work has examined the link between expressed trunk check behaviors and reproductive hormones. Slade-Cain et al. 
(2008) found the rate of trunk investigations was associated with changes in estrous state. However, to our knowledge, ours is the first study to evaluate the use of trunkcheck behaviors to detect parturition status via chemo-signaling. Our results are very promising in that multiple trunk-check behaviors were found to vary with the parturition status of the female as signaled by P4 and/or cortisol.

\section{Mammary Glands and Progesterone}

Both Mammary Gland Self-Checks and Other-Checks from herd mates increased in occurrence with decreasing levels of P4. As discussed previously, I suggested that selftrunk-check behavior is more likely due to physical changes in the body that may be mediated by hormones, while other trunk-check behavior from herd mates is more likely due to chemo-signaling and direct sensing of hormones.

Mammary glands undergo significant development in pregnancy, with ductal and secretory tissue mass increasing throughout pregnancy (Senger 2003). In a number of mammal species, progesterone is a main driver of final alveolar tissue development in late gestation. Brown et al. (2004a) performed an ultrasound on mammary tissue after the observed P4 drop, and noted increased fluid in milk channels, enlarged ducts, and defined glandular tissue. These physical changes to mammary tissue may initiate selftrunk-check behavior in late gestation.

Birth protocols suggest that colostrum may begin dripping from the mammary gland in the days and hours preceding parturition, though it is not apparent in all cases. Brown et al. (2004a) noted milk staining the front legs of an Asian elephant after the P4 
drop was observed. If present, herd mates may use colostrum or milk to detect changes in hormones that are present in the secretions. Other hormones involved in the synthesis of milk, including prolactin, and placental lactogen (Senger 2003), may also act as chemo-signals in the mammary tissue. We did not evaluate these hormones, but recommend further study.

Vulva, Progesterone and Cortisol

Vulva Self-Checks increased in occurrence with decreasing levels of P4 and increasing levels of cortisol. Self-checks of the vulva are likely related to physical changes in the ano-genital region. An increase in mucous from the vulva has been observed between 1-3 days preparturition (Szdzuy et al. 2006). Previous work has also found an increase in tail beating across the vulva prior to parturition (Szdzuy et al. 2006). It was suggested that tail beating occurred due to swelling observed in the anogenital region, an observation echoed in birth protocols. The vulva self-checks observed in our study may also be due to similar physical changes in mucous and/or swelling in the region. These physical changes may be associated with the decreasing levels of P4 and increasing levels of cortisol that occur in late gestation, or possibly with another hormone that was not evaluated in this study.

\section{Anus and Cortisol}

The ano-genital region is one of the most commonly checked areas by herd mates (Rasmussen \& Schulte 1998). I report anus checks from herd mates increased in occurrence with increasing levels of cortisol. Glucocorticoid metabolites are present in 
fecal matter and are used as a measure of stress response (Brown 2000; Shepherdson et al. 2004; Wielebnowski \& Watters 2007; Shepherdson et al. 2013). In elephants, cortisol surges are common in late gestation, as well as on the day of parturition (Meyer et al. 2004), and herd mates may be able to detect the presence of glucocorticoid metabolites associated with these surges in the anal region.

GLMM that included P4 levels were also significant, but the cortisol model was ultimately a better fit after stepwise reduction. However, a closer examination of the role of P4 and anus trunk-checks is warranted. Female elephants are known to regularly trunk-check the ano-genital region of female herd mates to detect estrous state (Rasmussen \& Schulte 1998), and they show a heightened response during the recipient's follicular phase (Rasmussen \& Schulte 1998), a time with low progesterone levels. Given the drop in P4 that occurs just prior to parturition, herd mates may be detecting low levels of progesterone in late gestation similar to that detected in the follicular phase of the estrous cycle.

Ear/Temporal Gland

No significant models were found that used either Self- or Other- Ear TrunkCheck. According to Schulte \& Rasmussen (1999), female Asian elephants rarely secrete from their temporal glands, though it has been observed in some cases (Brown et al. 2004a). If secretions are not present, there may be little or no gestation- or parturitionrelated hormones for herd mates to detect via chemo-signaling. In the absence of 
secretions, it is also unlikely that there are physical ear or temporal-gland changes that would be associated with self-checks of this region.

The significant models for herd-mate trunk-check behavior towards the dam (mammary gland and anus) suggest that chemo-signaling may play an important role in herd-mate detection of parturition-related hormones. I previously discussed the benefits of hiding parturition, especially from possible predators. Here I suggest that inter-individual signaling among elephants may be another evolutionarily sound practice in the wild. Female Asian elephants in the wild form groups of related individuals (Vidya \& Sukumar 2005). Thus, a new calf is related to all the other individuals in the group. Protection of the calf is therefore advancing shared genes among the herd. Protection of a new calf starts at birth, with the herd surrounding the laboring mother and eventually the newborn calf (Schulte 2000). Females in the group who are not currently reproductive are known to take on an important allomothering or "auntie" role, helping to care for another female's young (Schulte 2000). Having a method to alert herd mates to the imminent arrival of the calf directly benefits the calf by garnering protection from the herd, and ultimately benefits the entire herd by protecting their shared genes.

Although herd structure in captivity differs from that observed in the wild in that most individuals are not related, I would argue that the same mechanisms are at play. It has been shown that elephants from captive or semi-captive environments that are reintroduced to the wild are less likely to be related, but are known to establish social 
groups (Thitaram et al. 2015). In addition, allomothering roles have been observed in captive herds (Schulte 2000).

Finally, it is important to note possible limitations to this study. Our results may be confounded by normally existing behaviors. For example, body movements and "trunk-to" behaviors are correlated with elephant rank. Matriarch females are more often on the receiving end of "trunk-to" behaviors (Esposito 2008). In addition, the parity history of each dam may also confound results, with more experienced females behaving differently than nulliparous females. Future research should compare the behaviors between Rose Tu's first and second pregnancies, along with other females with multiple pregnancies.

While I found a variety of promising behavior changes in the final weeks of gestation, some of these changes may have already been underway when our behavioral data collection began. Such behavioral changes would have been missed by our analyses. Future research should consider collecting behavioral data prior to the 4 or 5 weeks before parturition that we monitored, and also comparing data of the same individuals when pregnant versus not pregnant

Our strongest recommendation is that institutions provide space for the pregnant dam to walk backwards, both in the daytime and at night. In addition, providing elevated substrate may be useful for dams seeking to raise her hind quarters to alleviate pressure on the pelvic rim or allow the calf to optimize position prior to parturition. 


\section{References}

Bardi, Shimizu, Barrett, Huffman, \& Borgognini-Tarli. (2003). Differences in the endocrine and behavioral profiles during the peripartum period in macaques. Physiology \& Behavior, 80(2), 185-194.

Blogg, Russell W., "Patterns of Chemosensory Behavior in a Closed Population of Wild African Elephants (Loxodonta Africana)" (2012). Electronic Theses \& Dissertations. 15. http://digitalcommons.georgiasouthern.edu/etd/15

Brown, J. (2000). Reproductive endocrine monitoring of elephants: An essential tool for assisting captive management. Zoo Biology, 19(5), 347-367.

Brown, J., Göritz, F., Pratt-Hawkes, N., Hermes, R., Galloway, M., Graham, L., . . . Hildebrandt, T. (2004a). Successful artificial insemination of an Asian elephant at the National Zoological Park. Zoo Biology, 23(1), 45-63.

Brown, J. L., \& Lehnhardt, J. (1995). Serum and urinary hormones during pregnancy and the peri-and postpartum period in an Asian elephant (Elephas, maximus). Zoo Biology, 14(6), 555-564.

Brown, J. L., Olson, D., Keele, M., \& Freeman, E. W. (2004b). Survey of the reproductive cyclicity status of Asian and African elephants in North America. Zoo Biology, 23(4), 309-321.

Brown, J. L., Schmitt, D. L., Bellem, A., Graham, L. H., \& Lehnhardt, J. (1999). Hormone Secretion in the Asian Elephant (Elephas maximus): Characterizationof Ovulatory and Anovulatory Luteinizing Hormone Surges.Biology of Reproduction, 61(5), 1294-1299.

Buford, J., Zernicke, R., \& Smith, J. (1990). Adaptive control for backward quadrupedal walking. I. Posture and hindlimb kinematics. Journal of Neurophysiology, 64(3), 745-55.

Challis, Sloboda, Matthews, Holloway, Alfaidy, Howe, ... Newnham. (2000). Fetal hypothalamic-pituitary adrenal (HPA) development and activation as a determinant of the timing of birth, and of postnatal disease. Endocrine Research, 26(4), 489-504.

Chaloupková, H., Illmann, G., Neuhauserová, K., Simecková, M., \& Kratinová, P. (January 01, 2011). The effect of nesting material on the nest-building and maternal behavior of domestic sows and piglet production. Journal of Animal Science, 89, 2, 531-7. 
Dale, R., Soltis, Joseph, \& Brown, Janine L. (2010). Birth statistics for African (Loxodonta africana) and Asian ( Elephas maximus ) elephants in human care: History and implications for elephant welfare. Zoo Biology, 29(2), 87-103.

De Silva, S., Ranjeewa, A., \& Kryazhimskiy, S. (2011). The dynamics of social networks among female Asian elephants. BMC Ecology, 11, 17.

Dehnhard, M., Hatt, J. M., Eulenberger, K., Ochs, A., \& Strauss, G. (2003). Headspace solid-phase microextraction (SPME) and gas chromatography-mass spectrometry (GC-MS) for the determination of $5 \alpha$-androst-2-en-17-one and$17 \beta$-ol in the female Asian elephant: Application for reproductive monitoring and prediction of parturition. The Journal of steroid biochemistry and molecular biology, 84(2), 383-391.

Dunbar, R.I.M. (1976). Some Aspects of Research Design and Their Implications in the Observational Study of Behaviour. Behaviour, 58(F0020001), 78-98.

Esposito, Rhea M. (2008) Dissertation: Effect of Matriarchs on Group Interactions, Kinship Fitness, and Differences In Chemosensory Behavior of African Elephants (Loxodonta Africana). Georgia Southern University.

Fanson, K. V., Keeley, T., \& Fanson, B. G. (2014). Cyclic changes in cortisol across the estrous cycle in parous and nulliparous Asian elephants. Endocrine connections, 3(2), 57-66.

Fernando, P., \& Lande, R. (2000). Molecular genetic and behavioral analysis of social organization in the Asian elephant ( Elephas maximus). Behavioral Ecology and Sociobiology, 48(1), 84-91.

Glaeser, S. S., Hunt, K. E., Martin, M. S., Finnegan, M., \& Brown, J. L. (2012). Investigation of individual and group variability in estrous cycle characteristics in female Asian elephants (Elephas maximus) at the Oregon Zoo. Theriogenology, 78(2), 285-296.

González-Mariscal, G., Díaz-Sánchez, V., Melo, A. I., Beyer, C., \& Rosenblatt, J. S. (January 01, 1994). Maternal behavior in New Zealand white rabbits: quantification of somatic events, motor patterns, and steroid plasma levels. Physiology \& Behavior, 55, 6, 1081-9

Hartsock, T., \& Barczewski, R. (1997). Prepartum behavior in swine: Effects of pen size. Journal of Animal Science, 75(11), 2899-904.

Hess, D L, Schmidt, A M, \& Schmidt, M J. (1983). Reproductive cycle of the Asian elephant (Elephas maximus) in captivity. Biology of Reproduction, 28(4), 767-73. 
Hildebrandt, T., Göritz, F., Hermes, R., Reid, C., Dehnhard, M., \& Brown, J. (2006). Aspects of the reproductive biology and breeding management of Asian and African elephants Elephas maximus and Loxodonta africana. International Zoo Yearbook, 40(1), 20-40

Hodges,J.K., Fieb,M., and Heistermann,M. 1999. Non-Invasive Reproductive Assessment in the African Elephant: Applications to Studies in Captivity and in the Wild. Seventh World Conference on Breeding Endangered Species: Linking Zoo and Field Research to Advance Conservation.

Hodges, J. K., Henderson, C., \& McNeilly, A. S. (1983). Circulating oestrogen concentrations during pregnancy in the African elephant (Loxodonta africana). Journal of reproduction and fertility, 67(1), 121-127.

Horback, K. M., Miller, L. J., Andrews, J. R., \& Kuczaj, S. A. (2014). Diurnal and nocturnal activity budgets of zoo elephants in an outdoor facility. Zoo biology, 33(5), 403410.

Hutchins, M., \& Keele, M. (2006). Elephant importation from range countries: Ethical and practical considerations for accredited zoos. Zoo Biology, 25(3), 219-233.

Ingram, J. N., Shaw, G., \& Renfree, M. B. (1999). Cortisol in fetal fluids and the fetal adrenal at parturition in the tammar wallaby (Macropus eugenii).Biology of reproduction, 60(3), 651-655.

Kajaysri, J., \& Nokkaew, W. (2014). Assessment of pregnancy status of Asian elephants (Elephas maximus) by measurement of progestagen and glucocorticoid and their metabolite concentrations in serum and feces, using enzyme immunoassay (EIA). The Journal of Veterinary Medical Science,76(3), 363.

Kattesh, H. G., Baumbach, G. A., Gillespie, B. B., Schneider, J. F., \& Murai, J. T. (1997). Distribution between protein-bound and free forms of plasma cortisol in the gilt and fetal pig near term. Neonatology, 72(3), 192-200.

Keele, 2015: North America Asian Elephant Studbook: http://www.elephanttag.org/professional/AsianElephantNAStudbook2014.pdf

Kowalski, N., Dale, R., Mazur, C., Soltis, Joseph, \& Brown, Janine L. (2010). A survey of the management and development of captive African elephant ( Loxodonta africana ) calves: Birth to three months of age. Zoo Biology, 29(2), 104-119.

Lang, E. (1967). The birth of an African elephant Loxodonta africana at Basle Zoo.International Zoo Yearbook, 7(1), 154-157. 
Leger, D. W. (1977). An empirical evaluation of instantaneous and one-zero sampling of chimpanzee behavior. Primates, 18(2), 387-393.

Leuthold, W., \& Leuthold, B. M. (1975). Parturition and related behaviour in the African elephant. Zeitschrift für Tierpsychologie, 39(1-5), 75-84.

Lueders, I., Drews, B., Niemuller, C., Gray, C., Rich, P., Fickel, J., ... \& Hildebrandt, T. B. (2010). Ultrasonographically documented early pregnancy loss in an Asian elephant (Elephas maximus). Reproduction, Fertility and Development, 22(7), 1159-1165.

Lueders, I., Niemuller, C., Rich, P., Gray, C., Hermes, R., Goeritz, F., \& Hildebrandt, T. B. (2012). Gestating for 22 months: luteal development and pregnancy maintenance in elephants. Proceedings of the Royal Society of London B: Biological Sciences, 279(1743), 3687-3696.

Maberry, M. (1963). BREEDING INDIAN ELEPHANTS Elephas maximus AT PORTLAND ZOO. International Zoo Yearbook, 4(1), 80-83.

Martin, P., \& Bateson, P. P. G. (2007). Measuring behaviour : An introductory guide (3rd ed.). Cambridge ; New York: Cambridge University Press.

Meyer, Walker, Freeman, Steinetz, \& Brown. (2004). Species and fetal gender effects on the endocrinology of pregnancy in elephants. General and Comparative Endocrinology, 138(3), 263-270.

Moss, C. (1988). Elephant memories : Thirteen years in the life of an elephant family. New York: William Morrow and Company.

Negatu, \& Mcnitt. (2002). Hormone profiles and nest-building behavior during the periparturient period in rabbit does. Animal Reproduction Science, 72(1), 125135.

Oliveira, C. A. D., Felippe, E. C. G., \& Chelini, M. O. M. (2008). Serum cortisol and progestin concentrations in pregnant and non-pregnant Asian elephants (Elephas maximus). Research in veterinary science, 84(3), 361-363.

Olsen, J. H., Chen, C. L., Boules, M. M., Morris, L. S., \& Coville, V. R. (1994). Determination of reproductive cyclicity and pregnancy in elephant: luteinising hormone and progesterone cycles in African (Loxodonta africana) and Asian (Elephas maximus) elephants. Biol. Reprod, 38, 309-334.

Owen-Smith, R. (1988). Megaherbivores: The influence of very large body size on ecology (Cambridge studies in ecology). Cambridge ; New York: Cambridge University Press. 
Payne, K., Langbauer, B., \& Thomas, W. (1986). Infrasonic calls of the Asian elephant ( Elephas maximus ). Behavioral Ecology and Sociobiology, 18(4), 297-301.

Rasmussen, L., Schmidt, M., Henneous, R., Groves, D., \& Daves, G. (1982). Asian Bull Elephants: Flehmen-Like Responses to Extractable Components in Female Elephant Estrous Urine. Science, 217(4555), 159-162.

Rasmussen, L. E. L., \& Schulte, B. A. (1998). Chemical signals in the reproduction of Asian (Elephas maximus) and African (Loxodonta africana) elephants. Animal reproduction science, 53(1), 19-34.

Rees, P. (2003). Asian elephants in zoos face global extinction: Should zoos accept the inevitable? Oryx, 37(1), 20-22.

Rees, P. A. (2009). Activity budgets and the relationship between feeding and stereotypic behaviors in Asian elephants (Elephas maximus) in a zoo.Zoo biology, 28(2), 79-97.

Rees, L. H., Jack, P. M. B., Thomas, A. L., \& Nathanielsz, P. W. (1975). Role of foetal adrenocorticotrophin during parturition in sheep.

Ren, Lei, Butler, Melanie, Miller, Charlotte, Paxton, Heather, Schwerda, Delf, Fischer, Martin S., \& Hutchinson, John R. (2008). The movements of limb segments and joints during locomotion in African and Asian elephants.(Author abstract)(Report). Journal of Experimental Biology, 211(17), 2735.

Rietkerk, F., Hiddingh, H., \& Dijk, S. (1993). Hand-rearing an Asian elephant Elephas maximums at the Noorder Zoo, Emmen. International Zoo Yearbook,32(1), 244252.

Schaftenaar, W. (2013). Delayed postpartum fetotomy in an Asian elephant (Elephas maximus). Journal of Zoo and Wildlife Medicine : Official Publication of the American Association of Zoo Veterinarians, 44(1), 130-5.

Schaftenaar, W., Hildebrandt, T. B., \& Elephant, T. A. G. (2006). Veterinary guidelines for reproduction-related management in captive female elephants. Elephant TAG Veterinary Advisors.

Schmidt, M. J. (January 01, 1982). Studies on Asian elephant reproduction at the Washington Park Zoo. Zoo Biology, 1, 2, 141-147.

Schulte, B. A., \& Rasmussen, L. E. L. (1999). Signal-receiver interplay in the communication of male condition by Asian elephants. Animal Behaviour,57(6), 1265-1274. 
Schulte, B. A. (2000). Social structure and helping behavior in captive elephants. Zoo Biology, 19(5), 447-459.

Senger, P. (2003). Pathways to pregnancy and parturition (2nd ed.). Pullman, WA: Current Conceptions.

Shepherdson, D., Carlstead, K., \& Wielebnowski, N. (2004). Cross-institutional assessment of stress responses in zoo animals using longitudinal monitoring of faecal corticoids and behaviour. Animal Welfare, 13, S105-S113.

Shepherdson, D., Lewis, K. D., Carlstead, K., Bauman, J., \& Perrin, N. (2013). Individual and environmental factors associated with stereotypic behavior and fecal glucocorticoid metabolite levels in zoo housed polar bears. Applied Animal Behaviour Science, 147(3), 268-277.

Slade, Schulte, \& Rasmussen. (2003). Oestrous state dynamics in chemical communication by captive female Asian elephants. Animal Behaviour, 65(4), 813819.

Slade-Cain, B., Rasmussen, L., \& Schulte, B. (2008). Estrous state influences on investigative, aggressive, and tail flicking behavior in captive female Asian elephants. Zoo Biology, 27(3), 167-180.

Styles, T. (1982). The birth and early development of an African elephant at the Metro Toronto Zoo. International Zoo Yearbook, 22(1), 215-217.

Sukumar, R. (1989). Ecology of the Asian elephant in southern India. I. Movement and habitat utilization patterns. Journal of Tropical Ecology, 5(1), 1-18.

Sukumar, R. (2003). The living elephants : Evolutionary ecology, behavior, and conservation. New York: Oxford University Press.

Sukumar, R. (2006). A brief review of the status, distribution and biology of wild Asian elephants Elephas maximus. International Zoo Yearbook, 40(1), 1-8.

Szdzuy, Dehnhard, Strauss, Eulenberger, \& Hofer. (2006). Behavioural and endocrinological parameters of female African and Asian elephants Loxodonta africana and Elephas maximus in the peripartal period. International Zoo Yearbook,40(1), 41-50.

Taylor, V., \& Poole, T. (1998). Captive breeding and infant mortality in Asian elephants: A comparison between twenty western zoos and three eastern elephant centers. Zoo Biology, 17(4), 311-332.

The IUCN Red List of Threatened Species. Version 2016-1. <www.iucnredlist.org>. Downloaded on 29 June 2016. 
Thitaram, Dejchaisri, Somgird, Angkawanish, Brown, Phumphuay, ... Kangwanpong. (2015). Social group formation and genetic relatedness in reintroduced Asian elephants (Elephas maximus) in Thailand. Applied Animal Behaviour Science, 172, 52-57.

Thitaram, Pongsopawijit, Thongtip, Angkavanich, Chansittivej, Wongkalasin, . . . Mahasavankul. (2006). Dystocia following prolonged retention of a dead fetus in an Asian elephant ( Elephas maximus). Theriogenology, 66(5), 1284-1291.

Thuppil, V., \& Coss, R. (2013). Wild Asian elephants distinguish aggressive tiger and leopard growls according to perceived danger. Biology Letters, 9(5), 20130518.

U.S. Fish Wildlife Service. Division of International Conservation. (2002). Asian Elephant Conservation Act : Summary report 1999-2001. Washington, D.C.]: [U.S. Dept. of the Interior, U.S. Fish \& Wildlife Service, Division of International Conservation].

Vidya, T., \& Sukumar, N. (2005). Social organization of the Asian elephant ( Elephas maximus ) in southern India inferred from microsatellite DNA. Journal of Ethology, 23(2), 205-210.

Wielebnowski, N., \& Watters, J. (2007). Applying fecal endocrine monitoring to conservation and behavior studies of wild mammals: Important considerations and preliminary tests. Israel Journal Of Ecology \& Evolution, 53(3-4), 439-460.

Wiese, R., \& Brown, Janine L. (2000). Asian elephants are not self-sustaining in North America. Zoo Biology, 19(5), 299-309.

Wiese, R., \& Willis, K. (2004). Calculation of longevity and life expectancy in captive elephants. Zoo Biology, 23(4), 365-373.

Willis, Erin L., Kersey, David C., Durrant, Barbara S., \& Kouba, Andrew J. (2011). The Acute Phase Protein Ceruloplasmin as a Non-Invasive Marker of Pseudopregnancy, Pregnancy, and Pregnancy Loss in the Giant Panda. PLoS ONE, 6(7), E21159.

Yamamoto, Y., Yamamoto, T., Taya, K., Watanabe, G., Stansfield, F. J., \& Allen, W. R. (2011). Placentation in the African elephant (Loxodonta africana). V. The trophoblast secretes placental lactogen. Placenta, 32(7), 506-510.

Yamamoto, Y., Yuto, N., Yamamoto, T., Kaewmanee, S., Shiina, O., Mouri, Y., ... \& Watanabe, G. (2012). Secretory pattern of inhibin during estrous cycle and pregnancy in African (Loxodonta africana) and Asian (Elephas maximus) elephants. Zoo biology, 31(5), 511-522. 